\title{
2 Nachhaltiges Wirtschaften und Unternehmensmanagement
}

\section{1 Ökonomie natürlicher und gesellschaftlicher Ressourcen}

\author{
Johannes Schmidt und Sebastian Wehrle
}

Institut für Nachhaltige Wirtschaftsentwicklung,

Department für Wirtschafts- und Sozialwissenschaften (WiSo)

johannes.schmidt@boku.ac.at, sebastian.wehrle@boku.ac.at

\subsubsection{Einleitung}

Bei der Produktion und dem Konsum von Gütern und Dienstleistungen machen wir vielfältigen Gebrauch von Ressourcen und Umwelt, etwa indem wir Erdölprodukte verbrennen oder Fischfang betreiben. Die Nutzung von Ressourcen sowie der Eintrag von Schadstoffen und Abfällen in die Umwelt beeinflussen langfristig die Ressourcenverfügbarkeit und die Umweltqualität. Die Umwelt- und Ressourcenökonomie untersucht diese Beziehungen: Die Nutzung von Umwelt und Ressourcen wird hinsichtlich ihrer Effizienz und Verteilungswirkungen analysiert. Das Fach ist stark interdisziplinär geprägt und vermittelt neben sozialen, technischen und ökonomischen Zusammenhängen auch ein Verständnis für die Wechselwirkungen zwischen Menschen und Ökosystemen. Diese Erkenntnisse bilden die Grundlage für die Bewertung von Regulierungsinstrumenten mit dem Ziel, die soziale Wohlfahrt zu erhöhen. Im Allgemeinen setzt die neoklassische Ökonomie dabei voraus, dass bestimmte Institutionen, also Regeln des Zusammenlebens (z.B. Eigentumsrechte, Märkte, staatliche Restriktionen und informelle Regeln im Handel), vorhanden sind und funktionieren. Die Neue Institutionenökonomik beschäftigt sich damit, wie solche Institutionen ausgestaltet sind bzw. werden sollen und welchen Einfluss sie auf unser Wirtschaften haben.

Umwelt- und Ressourcenökonominnen und -ökonomen beschäftigen sich neben mikroökonomischen Fragestellungen (bei denen Konsumentinnen und Konsumenten bzw. Produzentinnen und Produzenten im Mittelpunkt stehen) auch mit makroökonomischen Fragestellungen (welche die Wirtschaft als Ganzes betreffen), wie etwa der Frage nach der Möglichkeit nachhaltigen Wirtschaftswachstums.

Thomas Malthus, ein Vorreiter in Fragen des nachhaltigen Wachstums, beschäftigte sich bereits 1798 im „Essay on the Principle of Population“ (Malthus 1998) mit der Nutzung natürlicher Ressourcen durch den Menschen. Er postulierte, dass das lineare 


\section{Nachhaltiges Wirtschaften \& Unternehmensmanagement}

Wachstum landwirtschaftlicher Produktivität nicht mit dem exponentiellen Bevölkerungswachstum mithalten könne. Diese zwei unterschiedlichen Wachstumsprozesse müssten immer wieder in Einklang gebracht werden, weil die Bevölkerung ausreichend Nahrung benötigt. Laut Malthus muss dieser Prozess zwangsläufig zu wiederkehrenden Hungerkatastrophen führen. Er konnte nicht voraussehen, dass der rasante technische Fortschritt (z.B. in der Herstellung von synthetischen Stickstoffdüngern und Pestiziden, in der Züchtung von Nutzpflanzen und Nutztieren, in der Entwicklung moderner Maschinen sowie in der Intensivierung und Ausweitung der agrarischen Produktion auf neue, zuvor naturbelassene Flächen) eine immense Steigerung der globalen landwirtschaftlichen Produktion ermöglichte. So ernährt die Landwirtschaft heute weltweit sieben Mal so viele Menschen wie im 18. Jahrhundert und befriedigt ebenfalls einen immens gestiegenen Fleischkonsum. ${ }^{1}$ Gleichzeitig brachte diese Ausweitung und Intensivierung der landwirtschaftlichen Produktion neue Umweltprobleme mit sich (siehe Modell der Risikospirale, Abbildung 3.4.2). ${ }^{2}$

Moderne umwelt- und ressourcenökonomische Modelle beschreiben die Prozesse von Produktion und Konsum detaillierter: Vorhandene natürliche Ressourcen werden unter Einsatz von Kapital und Arbeit in Konsum- und Kapitalgüter transformiert, wobei auch Nebenprodukte wie Emissionen und Reststoffe erzeugt werden. Welche Relationen von Eingangs- und Reststoffen sind dabei aber effizient? Welche Rolle spielt technischer Fortschritt in diesen Prozessen? Wie viel Wachstum in der Bevölkerung und in anthropologisch geschaffenem Kapital ${ }^{3}$ ist möglich, ohne zukünftige Generationen einzuschränken bzw. den Zustand der heutigen Umwelt zu verschlechtern? Die Umweltund Ressourcenökonomie kann hier Beiträge zur Zielerreichung leisten, aber auch Synergien und Konflikte zwischen verschiedenen Zielen, wie zwischen SDG 8 (menschenwürdige Arbeit und Wirtschaftswachstum) und SDG 13 (Maßnahmen zum Klimaschutz) aufzeigen.

1 Die Produktion von Fleisch und anderen tierischen Produkten erfolgt mit einer deutlich geringeren Flächeneffizienz als jene pflanzlicher Lebensmittel (Wirsenius et al. 2010).

2 Im Zeitraum 1961-2012 stieg die Nahrungsmittelverfügbarkeit pro Kopf weltweit (FAO 2015), obwohl diese in einzelnen Ländern zurückging (z.B. Afghanistan, Somalia). Floud et al. (2011) zeigten für einige europäische Länder den Anstieg der Nahrungsmittelverfügbarkeit von $2.000 \mathrm{kcal} / \mathrm{Kopf} / \mathrm{Tag}$ im Jahr 1800 (ca. zu der Zeit, als Malthus sein Buch publizierte) auf über $3.600 \mathrm{kcal} / \mathrm{Kopf} / \mathrm{Tag} \mathrm{im}$ Jahr 2000. Die durchschnittliche Nahrungsmittelverfügbarkeit kann allerdings ein irreführender Indikator sein, wenn das Einkommen innerhalb einer Region ungleich verteilt ist. Deswegen sind weltweit noch immer ca. 800 Mio. Menschen unterernährt (1990 waren es noch rund 1 Mrd.) (FAO et al. 2015). Gleichzeitig hat sich in der Periode 1850-2015 die weltweite Waldfläche um 17\% verringert (Houghton und Nassikas 2017), und v.a. wegen des Einsatzes von mineralischen Kunstdüngern in der Landwirtschaft wurden die sicheren Grenzen der Stickstoffemissionen global bereits überschritten (Steffen et al. 2015).

3 Das anthropogen geschaffene Kapital umfasst physisches Kapital (z.B. Maschinen, Gebäude, Infrastruktur), intellektuelles Kapital (d.s. verkörperte Fähigkeiten und Wissen, die das Produktivitätspotenzial erhöhen) und soziales Kapital (d.s. nicht verkörperte Fähigkeiten und Wissen, wie z.B. Bücher und andere kulturelle Gebilde, die über die Zeit im sozialen Lernprozess weitergegeben und entwickelt wurden). 


\subsubsection{Wachstum und Nachhaltigkeit}

Einfache neoklassische Wachstumsmodelle zeigen, dass selbst ohne Berücksichtigung endlicher, natürlicher Ressourcen Wachstum nur begrenzt möglich ist. Um die Produktion des Wirtschaftssystems zu steigern, muss physisches Kapital (Fabriken, Straßen usw.) aufgebaut werden. Ab einem bestimmten Punkt übersteigen aber die Erhaltungskosten für das Kapital das Potenzial für Neuinvestitionen. Es kommt zu einem wachstumslosen Gleichgewicht, falls die Bevölkerung nicht wächst. Erst die Einführung von technologischem Fortschritt erlaubt weiteres Wachstum - allerdings unter Einschränkungen, weil auch Forschung und Entwicklung Ressourcen verbrauchen (Romer 1990).

Durch den aufsehenerregenden Bericht des Club of Rome in den 1970er-Jahren, „Limits to Growth“ (Meadows et al. 1972), haben die Wirtschaftswissenschaften die Bedeutung nachhaltigen Wirtschaftens angesichts der Endlichkeit natürlicher Ressourcen verstärkt aufgegriffen. In der Folge wurden Wirtschaftsmodelle mit Ressourcen- und Umweltkomponenten erweitert. So hat u.a. Robert Solow (1974) gezeigt, dass die Verwendung endlicher natürlicher Ressourcen, wie fossiler Energieträger, langfristig nicht zum Kollaps von Wirtschaftssystemen führen muss: Ist die Substitution von natürlichen Ressourcen und von anthropologisch geschaffenem Kapital grundsätzlich möglich, kann das Wirtschaftssystem - im Prinzip unendlich lange - konstanten Output produzieren. Als Beispiel kann die Transformation des Energiesystems von fossilen hin zu nichtfossilen Energieträgern dienen (siehe dazu auch Beitrag 5.1 und vgl. SDG 7 - bezahlbare und saubere Energie): Wenn natürliche Ressourcen wie Erdöl langfristig durch anthropologisch geschaffenes Kapital wie Windturbinen oder Atomkraftwerke ersetzt werden und keine nichtenergetischen Restriktionen wie Land- oder Rohstoffmangel auftreten, muss das Produktionsniveau des Wirtschaftssystems nicht notgedrungen sinken (siehe Abbildung 2.1.1). Robert Solow war der Ansicht, dass es nicht Aufgabe heutiger Generationen sei, bestimmte natürliche Ressourcen zu schützen, sondern vielmehr Wirtschaftssysteme so zu gestalten, dass die

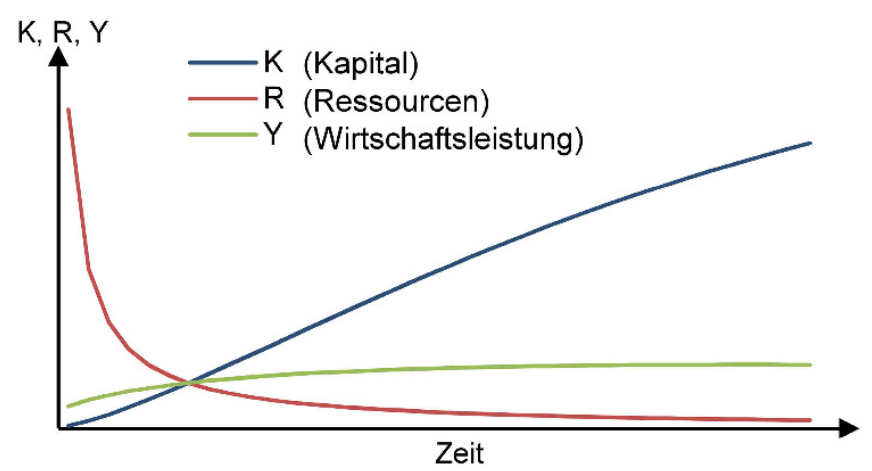

Abbildung 2.1.1: Nachhaltiges Wirtschaften mit endlichen Ressourcen 


\section{Nachhaltiges Wirtschaften \& Unternehmensmanagement}

Produktions- und Konsummöglichkeiten zukünftiger Generationen im Vergleich zu heutigen nicht eingeschränkt werden: In seiner Interpretation wirtschaften wir nachhaltig, wenn das Nutzennivau für zukünftige Generationen nicht sinkt.

Hartwick (1977) zeigte wenig später, dass Nachhaltigkeit langfristig nur zu erreichen ist, wenn die Knappheitsrente in den Aufbau von anthropologisch geschaffenem Kapital investiert wird. Die Knappheitsrente bezieht sich dabei auf ein Modell von Hotelling (1931). Er zeigte, dass die Preise von endlichen Ressourcen eine Rente aufweisen, die in der Regel über die Zeit wächst. ${ }^{4}$ Nach Hotelling ging man daher von über die Zeit wachsenden Preisen für endliche, natürliche Ressourcen aus. Empirische Untersuchungen konnten dazu keine eindeutigen Schlüsse liefern, da die Ergebnisse sehr vom Untersuchungszeitraum abhängen und die Preise für natürliche Ressourcen große Schwankungen aufweisen. ${ }^{5}$

Aus Hartwicks Regel zur Nachhaltigkeit folgt, dass der Aufbau eines langfristig nachhaltigen Produktions- und damit auch Konsumniveaus nur dann möglich ist (vgl. SDG 12 - verantwortungsvolle Konsum- und Produktionsmuster), wenn heutige Generationen auf einen Teil ihres möglichen Konsums verzichten. Des Weiteren müssten natürliche Ressourcen durch anthropologisch geschaffenes Kapital ersetzbar sein - und zwar relativ einfach: Um das Konsumniveau halten zu können, muss die Produktivität des anthropologisch geschaffenen Kapitals mindestens genauso hoch sein, wie jene der damit ersetzten natürlichen Ressourcen. So müssen für das Gelingen der globalen Energiewende z.B. nichtbrennstoffbasierte erneuerbare Energien (wie Windkraft oder Photovoltaik) mindestens so produktiv sein (also einen gleich hohen oder höheren Output pro eingesetzter Einheit Kapital und Arbeit haben) wie die Extraktion und Verwendung fossiler Energien. Außerdem sind Bevölkerungs- und Wirtschaftswachstum nur mit technologischem Fortschritt möglich, und die neuen Produktionstechnologien dürfen keine schweren Nebenfolgen verursachen (wie z.B. katastrophale Unfälle in der Atomkraft). Solow und Hartwick wenden das Konzept der schwachen Nachhaltigkeit an. Sie gehen davon aus, dass natürliche Ressourcen großteils durch anthropologisch geschaffenes Kapital ersetzt werden können. Bei Erdöl erscheint das einleuchtend. Jedoch geht

4 Im Vergleich zu Güterpreisen, die auf Wettbewerbsmärkten den marginalen Kosten der letzten produzierten Einheit entsprechen, sind die Preise für endliche Ressourcen höher als die marginalen Produktionskosten. Die Differenz wird als Knappheitsrente bezeichnet. Sie gibt die Opportunitätskosten des Verkaufs einer zusätzlichen Ressourceneinheit an und steigt, je knapper die Ressource wird. Die Entwicklung der Knappheitsrente mit der gleichen Rate des Marktzinssatzes wird als Hotelling-Regel bezeichnet.

5

Erweiterungen versuchen die Variabilität und den langfristigen Preisverlauf besser zu erklären (Perman et al. 2011). So hat die zunehmende Knappheit in bestimmten Ölfeldern (z.B. onshore) zu einem Preisanstieg geführt, der die Entwicklung und Verbreitung neuer Technologien (z.B. offshore, Schieferöle) ermöglichte. Aufgrund von Lerneffekten bei den Produzentinnen und Produzenten sinken die Kosten neuer Technologien im Laufe der Zeit jedoch, sodass die Fördermenge auch bei niedrigeren Preisen konstant bleiben kann. Auch Kartelle, unregelmäßige Explorationserfolge und geopolitische Ereignisse (wie z.B. Kriege) können den Preispfad kurz- und langfristig beeinflussen. 
man auch davon aus, dass natürliche Ressourcen wie die Klimaregulation durch anthropologisch geschaffenes Kapital (wie z.B. Klimaanlagen) ersetzt werden können.

Vertreterinnen und Vertreter einer starken Nachhaltigkeit widersprechen der vollständigen Ersetzbarkeit und argumentieren, dass natürliches und anthropologisch geschaffenes Kapital zum Großteil komplementär sind: Eine zu hohe Ausbeutung natürlicher Ressourcen würde zum Kollaps des Systems Erde führen. So entwickeln die Autorinnen und Autoren des Konzepts „Planetary Boundaries“ (Rockström et al. 2009) Schwellenwerte u.a. für die Menge an Treibhausgasemissionen oder den Verlust von Biodiversität (siehe Beiträge 4.1 und 4.4). Werden diese Schwellenwerte überschritten, ist die Stabilität der Ökosysteme so stark gefährdet, dass die entstehenden negativen Konsequenzen für den Menschen katastrophal und nicht mehr beherrschbar wären. So könnte eine vollständige Umstellung des Energiesystems auf erneuerbare Energieträger gravierende Konsequenzen für die Biodiversität haben und durch die Verfügbarkeit bestimmter endlicher Ressourcen - wie seltener Erden - begrenzt werden.

Starke Nachhaltigkeit kann in ökonomischen Modellen berücksichtigt werden (siehe Beitrag 6.1), indem die Substituierbarkeit mancher Ressourcen limitiert wird. Die Tragfähigkeit von natürlichen Systemen (D’Alessandro 2007) setzt dem Verbrauch von natürlichem Kapital ein Limit (z.B. absolute Treibhausgasemissionslimits). Weiteres Wirtschaftswachstum ist grundsätzlich auch unter der Komplementarität von natürlichem und anthropologisch geschaffenem Kapital vorstellbar, also unter absoluten Ressourcenlimits. Diese beruhen aber auf der Erwartung immerwährenden technologischen Fortschritts, der bei gesteigerter Produktivität geringere Emissionen, Abfälle und Schadstoffe erlauben müsste.

Wie wird nun Wachstum überhaupt gemessen? In vielen Modellen wird dazu das Bruttoinlandsprodukt (BIP; englisch Gross Domestic Product, GDP) verwendet. Dieses bezeichnet den Wert aller Güter und Dienstleistungen, die in einem Land innerhalb eines Jahres produziert werden. Die Verwendung des BIP als wirtschaftspolitische Zielgröße ist jedoch mit vielen Problemen behaftet: So werden im BIP nicht alle Tätigkeiten erfasst (z.B. unbezahlte Hausarbeit, Entnahme von Ressourcen), und umweltschädliche Wirtschaftstätigkeiten gehen positiv in das BIP ein. Umweltschäden und Ressourcenentnahme finden in einer umweltbezogenen volkswirtschaftlichen Gesamtrechnung - System of Environmental-Economic Accounting (SEEA) Berücksichtigung. In manchen Regionen liegt dieses „grüne“ BIP deutlich unter dem traditionellen BIP (Bartelmus 2009). Eine viel umfassendere Bewertung des Fortschritts von Volkswirtschaften fordern Stiglitz et al. (2018), da das BIP und auch Abwandlungen wie das grüne BIP nicht in der Lage sind, Lebensqualität, Lebenszufriedenheit und Glück zu messen. Sie plädieren für die Entwicklung eines Bündels 


\section{Nachhaltiges Wirtschaften \& Unternehmensmanagement}

an Indikatoren, die zeigen, wer vom Wachstum profitiert, ob Wachstum nachhaltig ist, wie Menschen ihre Lebensqualität einschätzen und welche Faktoren zum Erfolg eines Individuums oder eines Landes beitragen (siehe Fallbeispiel 2.1.1). Die Vermessung von Volkswirtschaften (z.B. hinsichtlich ihres Einflusses auf die Umwelt und die soziale Wohlfahrt, der Bestimmung von Bedingungen für ihre langfristig nachhaltige Entwicklung und der empirischen Analyse derselben) sind ein zentraler Bestandteil der Umwelt- und Ressourcenökonomie, genauso wie jene Faktoren, die die Ressourcenverwendung in Volkswirtschaften beeinflussen.

\section{Fallbeispiel 2.1.1: Messung des gesellschaftlichen Fortschritts}

Im Jahr 2013 gründete die OECD, die Organisation für wirtschaftliche Zusammenarbeit und Entwicklung, die „High Level Expert Group on the Measurement of Economic Performance and Social Progress", um Vorschläge zu evaluieren, wie man den gesellschaftlichen Fortschritt unabhängig vom BIP messen könnte. Die Vorsitzenden der Expertengruppe, Joseph Stiglitz, Jean-Paul Fitoussi und Martine Durand publizierten daraufhin im Jahr 2018 den Bericht „Beyond GDP: Measuring what counts for economic and social performance". Sie beschreiben, dass das BIP kein geeigneter Indikator sei, um soziale Wohlfahrt zu messen - wofür er auch nicht entwickelt wurde, sondern um konjunkturelle Wirtschaftsschwankungen und -zyklen zu messen. Eine starke wirtschaftspolitische Ausrichtung an das BIP ist daher nur eingeschränkt erfolgreich darin, die Gesamtwohlfahrt zu heben.

Stiglitz et al. (2018) nennen als Beispiel die Politikmaßnahmen, die infolge der Krise von 2008 getroffen wurden. Während die restriktiven Budgetmaßnahmen (teilweise) zu einem Ansteigen des BIP führten (in vielen Fällen wurde nicht einmal dieses Ziel erreicht), erhöhten sie gleichzeitig die ökonomische Unsicherheit für breite Teile der Bevölkerung, machten vielen jungen Menschen den Einstieg in das Erwerbsleben unmöglich (z.B. in Südeuropa) und zerstörten das Vertrauen in Institutionen, weil die Maßnahmen als unfair wahrgenommen wurden. Hätte sich die Politik an einem breiteren Indikatorenbündel orientiert (welches die wahrgenommene ökonomische Unsicherheit, die Verteilung von Einkommen, Vermögen und v.a. auch Chancen berücksichtigt sowie persönliches Wohlbefinden und die ökologischen Konsequenzen unseres Wirtschaftens miteinbezieht), wären mit hoher Wahrscheinlichkeit andere Maßnahmen getroffen worden. Stiglitz et al. (2018) betonen jedoch, dass sie nicht grundsätzlich wachstumskritisch sind, sondern das richtige Wachstum forcieren wollen.

Stiglitz et al. (2018) halten das Indikatorenbündel, welches für die nachhaltigen Entwicklungsziele (SDGs) entwickelt wurde, für zu umfangreich (mehr als 200 Indikatoren), um es in allen Ländern umfassend zu erheben und politische Maßnahmen daran auszurichten. Sie beschreiben in ihrem Bericht detailliert die Fortschritte in der Entwicklung solcher Indikatoren, noch vorhandene Lücken und welche Länder diese Indikatoren bereits erfolgreich anwenden.

Von der Diskussion um die Messung gesellschaftlichen Fortschritts inspiriert, publiziert Statistik Austria (s.a.) unter dem Titel „Wie geht's Österreich“ ein Bündel an Indikatoren. In Österreich ist z.B. das Haushaltseinkommen langsamer gestiegen als das BIP, und die Wohnkostenüberlastung hat in den letzten Jahren wieder zugenommen. Auch manche Umweltindikatoren, wie etwa die Treibhausgasemissionen und die verbauten Flächen, zeigen eine besorgniserregende Entwicklung. Gleichzeitig sind die Tötungsdelikte seit dem Jahr 2000 stark gefallen, haben sich die Einkommensunterschiede zwischen Männern und Frauen verringert, genauso wie die frühzeitige Sterblichkeit. Für die subjektiv wahrgenommene Zufriedenheit gibt es keine klaren Ergebnisse. Die Zeitreihe ist zu kurz, und es sind nur kleine Veränderungen beobachtet worden. 


\subsubsection{Marktversagen und Regulierung}

Die oben vorgestellten makroökonomischen Modelle sind stark vereinfachte Darstellungen von Volkswirtschaften. Einzelne Märkte, deren Interaktionen und darin auftretende Ineffizienzen werden nicht im Detail analysiert. Mikroökonomische Modelle beschäftigen sich mit genau dieser Fragestellung. Sie versuchen Instrumente bereitzustellen, die das Verhalten der Marktakteure steuern, um so mögliche Ineffizienzen (z.B. zu hohe Umweltverschmutzung) zu vermeiden. Hierfür steht ein breites umweltökonomisches Instrumentarium zur Verfügung - von Verboten bis zu Preisanpassungen und handelbaren Verschmutzungszertifikaten. Im Gegensatz zu Marketingansätzen, die versuchen, die Präferenzen von Individuen zu beeinflussen (z.B. die Neigung zu biologisch hergestellten Nahrungsmitteln über Werbung zu erhöhen), versuchen umweltökonomische Instrumente, die Anreize so anzupassen, dass Individuen in ihrem eigenen Interesse effiziente Konsum- oder Produktionsentscheidungen treffen (also z.B. durch die Erhöhung von Preisen von konventionell produzierten Produkten über Steuern oder auch durch das Verbot mancher Produktionsmittel, wie genverändertes Saatgut). Im Folgenden diskutieren wir zwei Fälle, in denen Märkte ineffiziente Allokationen erzeugen können: beim Auftreten von Externalitäten und in der Bereitstellung öffentlicher Güter. In beiden Fällen gibt es einige Optionen, um einem Marktversagen entgegenzuwirken.

\subsubsection{Externalitäten}

Viele unserer Produktions- und Konsumaktivitäten verursachen Emissionen. Diese reichern sich in der Umwelt an (z.B. Nitrat im Grundwasser, $\mathrm{CO}_{2}$ in der Atmosphäre, Schwermetalle im Boden), weil sie nur teilweise und langsam in unschädliche Formen umgewandelt werden. Die Überschreitung der natürlichen Absorptions- und Filterungskapazitäten der Umwelt kann zu Umweltschäden führen. Während Emissionen üblicherweise in physischen Einheiten gemessen werden (z.B. kg, t/ha, ppm), bewertet man Umweltschäden oft monetär (z.B. €). Umweltökonomische Analysen untersuchen den grundlegenden Zielkonflikt zwischen den Produktions- und Konsumaktivitäten und den Umweltschäden: Umweltverschmutzung zu vermeiden, ist zwar nützlich (z.B. Verbesserung der Luftqualität), es verursacht aber auch Kosten. Sind die (monetären) Umweltschäden und die Vermeidungskosten bekannt, lässt sich daraus das ökonomisch effiziente Verschmutzungsniveau bestimmen. Zur Implementierung eines gesellschaftlich optimalen Verschmutzungsniveaus steht eine Vielzahl von umweltpolitischen Instrumenten zur Verfügung (siehe Beitrag 3.2). Prinzipiell lässt sich eine Regulierung über Preise (z.B. Umweltsteuern), Mengen (z.B. Produktionsquoten), über eigens geschaffene Märkte für Verschmutzungszertifikate oder über Grenzwerte 


\section{Nachhaltiges Wirtschaften \& Unternehmensmanagement}

und Verbote ${ }^{6}$ gestalten. Zudem hat Ronald Coase (1960) gezeigt, dass effiziente Lösungen auch ohne staatlichen Eingriff möglich sind, sofern die Eigentumsrechte an Produktionsmitteln und Umweltgütern klar definiert und einer der Verhandlungsparteien zugeteilt sind. Die betroffenen Parteien können sich infolge auf eine gemeinsame Lösung einigen. Auf internationaler Ebene stellt die Verhandlungslösung die wichtigste Form der Umweltregulierung dar (z.B. Paris-Abkommen für den internationalen Klimaschutz im Jahr 2015, Montreal-Abkommen zum Schutz der Ozonschicht im Jahr 1989).

Die Besteuerung von Emissionen verteuert z.B. die emissionsintensive Produktion von Gütern. Der Anreiz, andere Produktionstechnologien zu wählen, die weniger Emissionen verursachen, wird erhöht. In letzter Zeit rückt auch die Verteilungswirkung solcher Instrumente in den Mittelpunkt der Forschung: Steuern wirken auf den Energiekonsum grundsätzlich regressiv. Haushalte mit niedrigem Einkommen werden also relativ stärker belastet als jene mit hohem Einkommen, sodass bei steigendem Einkommen die Ausgaben für Konsumgüter und Energie relativ sinken (Kirchner et al. 2019; Klenert et al. 2018). Die Umweltökonomie liefert Vorschläge, um diese negative Verteilungswirkung zu kompensieren. Einnahmen aus $\mathrm{CO}_{2}$-Steuern können z.B. umverteilt werden, um so den regressiven Effekt auszugleichen (für weitere Beispiele siehe Fallbeispiel 2.1.2). ${ }^{7}$

\subsubsection{2 Öffentliche Güter}

Öffentliche Güter werden anhand zweier Eigenschaften definiert: (1) Werden sie angeboten, ist es unmöglich, nichtzahlungsbereite Personen vom Konsum auszuschließen (Nichtausschließbarkeit), und (2) der Konsum einer Person schränkt den Konsum anderer Personen nicht ein (Nichtrivalität). Zu öffentlichen Gütern zählen u.a. Demokratie, Rechtstaatlichkeit, öffentliche Bildung und Forschung, Kultur, öffentliche Verwaltung, Gesundheits- und Sozialversorgung sowie innere und äußere Sicherheit. Der Bereitstellung öffentlicher Güter in Wirtschaftssystemen kommt eine besondere Bedeutung zu, da Märkte hier nicht oder nur sehr eingeschränkt funktionieren und die Bereitstellung dieser Güter daher gemeinschaftlich organisiert werden muss (in den meisten Fällen über staatliche Institutionen). Die Bereitstellung dieser Güter

${ }^{6}$ Grenzwerte und Verbote sind eher ineffizient, führen also zu höheren Umweltschutzkosten als notwendig. Gleichzeitig gelten sie aber als sehr effektiv und relativ einfach umzusetzen. Wie der Dieselskandal gezeigt hat, sind aber auch sie anfällig für Manipulationen.

7 Die Schweiz führte neben einer $\mathrm{CO}_{2}$-Steuer auch ein Kompensationssystem ein: Die Steuereinnahmen werden gleichverteilt auf alle Bürgerinnen und Bürger wieder gutgeschrieben (sogenannte lump-sum payments). Dadurch werden niedrige Einkommen im Vergleich zu hohen relativ entlastet. Der umweltpolitische Nachteil einer solchen Maßnahme ist, dass die eingenommenen Steuern nicht für andere Klimaschutzmaßnahmen, wie Investitionen in den öffentlichen Verkehr, zur Verfügung stehen. 


\section{Fallbeispiel 2.1.2: Umweltökonomie und die Energiewende}

Die Umstellung des globalen Energiesystems von fossilen Energieträgern auf solche mit wenigen oder gar keinen $\mathrm{CO}_{2}$-Emissionen ist ein wichtiger Beitrag zur Reduktion von Treibhausgasemissionen und damit zur Beschränkung der globalen Erwärmung. Eine Möglichkeit stellen erneuerbare Energien dar, wobei Photovoltaik (PV) als eine der wichtigsten erneuerbaren Zukunftstechnologien gilt. Die Umwelt- und Energieökonomie hat bedeutende Beiträge zum Verständnis der Entwicklung und des Einsatzes dieser Technologie geliefert. Dies wird hier exemplarisch an zwei Beispielen aufgezeigt.

\section{Logik von Einspeisetarifen}

Im Jahr 2000 wurden in Deutschland Einspeisetarife eingeführt - basierend auf der Beobachtung, dass eine neue Technologie, soll sie marktreif gemacht werden, einer Anstoßförderung bedarf. Der vermehrte Einsatz der Technologie entlang der gesamten Wertschöpfungskette soll Lerneffekte anregen, die zu einer starken Verbilligung der Technologie führen. Das Ziel ist, sie infolge ohne Förderungen am Markt einsetzen zu können. Kostensenkungen bei Kapazitätserhöhungen werden über sogenannte Lernraten empirisch abgeschätzt. Dazu sind Daten über die Kosten von Technologien und deren Anwendung notwendig. Die Lernrate gibt an, um wie viel sich eine Technologie verbilligt, wenn die installierte Kapazität dieser Technologie verdoppelt wird. Die Lernrate für PV liegt zwischen $18 \%$ und $28 \%$, d.h., eine Verdopplung der Menge an installierter PV-Kapazität hat in der Vergangenheit die Kosten um 18\% bis 28\% reduziert (Mauleón 2016).

Aus der Lernratentheorie kann abgeleitet werden, welche Technologien für öffentliche Subventionen infrage kommen: Diese sollten jung und relativ unausgereift sein, weil nur dann große Lerneffekte zu erwarten sind. Gleichzeitig sollte die Technologie nicht auf sehr knappen Rohstoffen basieren, bei denen eine erhöhte Nachfrage die Preise stark ansteigen lassen würde. Trotzdem ist unklar, ob bei sehr jungen Technologien starke Lerneffekte auftreten werden. Bei PV erzielte die Einspeisetarifpolitik große Erfolge, weil einige notwendige Bedingungen erfüllt waren und die Technologie durch die Skalierung billiger wurde: Als die Förderpolitik eingeführt wurde, war die Technologie am Markt noch wenig verbreitet. Infolge wurden signifikante Kostenreduktionen erreicht: Die Gestehungskosten einer Stromeinheit, erzeugt aus PV, sind zwischen 2000 und 2018 um einen Faktor 10 gefallen. Derzeit liegt der globale Anteil von PV bei unter $2 \%$, einige weitere Verdopplungen der Kapazität und damit verbundene weitere Kostensenkungen erscheinen möglich.

Biomassekraftwerke wurden ebenfalls über Einspeisetarife gefördert. Hier war aber bereits bei der Einführung abzusehen, dass keine signifikante Kostendegression zu erwarten ist: Die Verbrennung von Biomasse und die Stromerzeugung daraus ist eine gut bekannte Technologie. Große Technologiesprünge waren daher unwahrscheinlich. Biomassekraftwerke nutzen außerdem einen knappen Rohstoff. Die Nachfrageerhöhung ließ die Preise für Biomasse ansteigen. Biomassekraftwerke hatten also teilweise sogar mit Kostensteigerungen zu kämpfen. Die Förderung von Biomasse war wohl aus anderen Gründen opportun, nicht aber originär technologisch-ökonomisch motiviert.

\section{Ökonomik dezentraler Photovoltaik}

Eine zweite umweltökonomische Erkenntnis betrifft die Förderung von PV für Haushalte. Diese hat vielfältige ökonomische Effekte: So wurde die Einkommensungleichheit in Deutschland leicht erhöht, weil v.a. einkommensstarke Haushalte (mit Eigenheimen) in der Lage waren, PV einzusetzen, während einkommensschwächere Haushalte die Kosten dafür über die Ökostromumlage mittragen müssen (Grösche und Schröder 2014). Um diese regressive Verteilungswirkung abzuschwächen, müsste man die Ökostromumlage einkommensabhängig gestalten. Gleichzeitig könnten aber auch einkommensschwächere Haushalte in Mietwohnungen an der Energiewende partizipieren. Dafür müssten allerdings rechtliche Rahmenbedingungen geändert werden, was in Österreich erst vor Kurzem geschehen ist. Hier wird deutlich, wie wichtig eine interdisziplinäre Herangehensweise ist, die auch eine rechtswissenschaftliche Expertise mit einschließt. 


\section{Nachhaltiges Wirtschaften \& Unternehmensmanagement}

verursacht in der Regel auch hohe Kosten. Ökonominnen und Ökonomen beschäftigen sich daher u.a. mit der Frage, welche Menge an öffentlichen Gütern bereitgestellt werden soll - deren Finanzierung konkurriert schließlich um das öffentliche Haushaltsbudget. Da die Finanzierung und Bereitstellung von öffentlichen Gütern zwangsläufig Mittel zwischen Bürgerinnen und Bürgern umverteilt und die gesellschaftliche Partizipation über sie beeinflusst wird, sind gesellschaftliche und politische Debatten über deren Bereitstellung unverzichtbar.

Die Eigenschaft der Nichtausschließbarkeit öffentlicher Güter gibt Anreize für sogenanntes Trittbrettfahrerverhalten. Dabei wird weniger von einem öffentlichen Gut bereitgestellt, als ökonomisch effizient wäre, da die Trittbrettfahrer für den Konsum des öffentlichen Gutes nicht direkt bezahlen müssen. Ein Beispiel für ein Trittbrettfahrerproblem bei öffentlichen Gütern ist der Klimaschutz. Von einer Verringerung von Treibhausgasemissionen in Österreich oder in Europa profitieren Menschen weltweit. Niemand kann von den positiven Auswirkungen der Emissionsreduktion auf das Klimasystem ausgeschlossen werden. Die unmittelbaren Kosten tragen aber jene, die die Emissionen reduzieren. Manche Regierungen und Staaten fahren daher auf dem Trittbrett mit, indem sie aus dem Pariser Abkommen aussteigen bzw. es nicht ratifizieren.

\subsubsection{Fazit}

Die Beispiele zeigen, dass wir vielfältige Möglichkeiten haben, unsere Wirtschaftssysteme auszugestalten. Angesichts dessen ist die wichtigste Frage unserer Generation nicht ob, sondern wie die Menschheit langfristig leben will. Die Umwelt- und Ressourcenökonomie liefert wichtige Konzepte und Werkzeuge, um die Optionen und Konsequenzen auszuloten und zu bewerten. Dabei ist eine Zusammenarbeit mit den Natur- und Ingenieurwissenschaften von hoher Bedeutung, um die Möglichkeiten und Konsequenzen wirtschaftlichen Handelns für unser System Erde besser verstehen zu können (siehe Beitrag 4.1). Gleichzeitig bedarf es einer intensiven Auseinandersetzung mit den benachbarten Sozialwissenschaften, um die „blinden Flecken“ der Ökonomie - z.B. die Rolle von Macht in Entscheidungen und jene von Institutionen oder soziologische Aspekte des Umweltverhaltens (siehe Beiträge 3.2 und 3.3) - verstärkt zu berücksichtigen.

\section{Literatur}

Bartelmus, P. (2009): The cost of natural capital consumption: Accounting for a sustainable world economy. Ecological Economics, 68, 1850-1857. https://doi.org/10.1016/j.ecolecon.2008.12.011.

Coase, R. H. (1960): The problem of social cost. The Journal of Law \& Economics, 3, 1-44. http://www.jstor.org/stable/724810. 
D'Alessandro, S. (2007): Non-linear dynamics of population and natural resources: The emergence of different patterns of development. Ecological Economics, 62, 473-481. https://doi.org/10.1016/i.ecolecon.2006.07.008.

FAO (Food and Agriculture Organization of the United Nations) (2015): Food Balance Sheets. Available at: http://www.fao.org/faostat/en [accessed 12.4.2019]

FAO, IFAD, and WFP (Food and Agriculture Organization of the United Nations; International Fund for Agricultural Development; World Food Programme) (2015): The State of Food Insecurity in the World 2015. Meeting the 2015 international hunger targets: taking stock of uneven progress. Rome: FAO.

Floud, R., Fogel, R. W., Harris, B., and Hong, S. C. (2011): The Changing Body: Health, Nutrition, and Human Development in the Western World since 1700. Cambridge, New York: Cambridge University Press.

Grösche, P. and Schröder, C. (2014): On the redistributive effects of Germany's feed-in tariff. Empirical Economics, 46, 1339-1383. https://doi.org/10.1007/s00181-013-0728-z.

Hartwick, J. (1977): Intergenerational equity and the investing of rents from exhaustible resources. The American Economic Review, 67, 972-74. https://www.jstor.org/stable/1828079.

Hotelling, H. (1931): The economics of exhaustible resources. Journal of Political Economy, 39, 2, 137-175. http://dx.doi.org/10.1086/254195.

Houghton, R. A. and Nassikas, A. (2017): Global and regional fluxes of carbon from land use and land cover change 1850-2015. Global Biogeochemical Cycles, 31, 456-472. https://doi.org/10.1002/2016GB005546.

Kirchner, M., Sommer, M., Kratena, K., Kletzan-Slamanig, D., and Kettner-Marx, C. (2019): $\mathrm{CO}_{2}$ taxes, equity and the double dividend - Macroeconomic model simulations for Austria. Energy Policy, 126, 295-314. https://doi.org/10.1016/j.enpol.2018.11.030.

Klenert, D., Mattauch, L., Combet, E., Edenhofer, O., Hepburn, C., Rafaty, R., and Stern, N., (2018): Making carbon pricing work for citizens. Nature Climate Change, 8, 669. https://doi.org/10.1038/s41558-018-0201-2.

Malthus, T. (1998): An Essay on the Principle of Population. Electronic Scholarly Publishing Project.

Mauleón, I. (2016): Photovoltaic learning rate estimation: Issues and implications. Renewable and Sustainable Energy Reviews, 65, 507-524. https://doi.org/10.1016/j.rser.2016.06.070.

Meadows, D. H., Meadows, D. L., Randers, J., and Behrens III, W. W. (1972): The Limits to Growth. New York: Universe Books.

Perman, R., Ma, Y., McGilvray, J., Common, M. S., and Maddison, D. (2011): Natural Resource and Environmental Economics. 4th revised edition. Harlow, Essex, New York: Addison Wesley.

Rockström, J., Steffen, W., Noone, K., Persson, A., Chapin, F. S., Lambin, E. F., Lenton, T. M., Scheffer, M., Folke, C., Schellnhuber, H. J., Nykvist, B., Wit, C. A. de, Hughes, T., Leeuw, S. van der, Rodhe, H., Sörlin, S., Snyder, P. K., Costanza, R., Svedin, U., Falkenmark, M., Karlberg, L., Corell, R. W., Fabry, V. J., Hansen, J., Walker, B., Liverman, D., Richardson, K., Crutzen, P., and Foley, J. A. (2009): A safe operating space for humanity. Nature, 461, 472-475. https://doi.org/10.1038/461472a.

Romer, P. M. (1990): Endogenous technological change. Journal of Political Economy, 98, S71S102. https://www.jstor.org/stable/2937632.

Solow, R. M. (1974): Intergenerational equity and exhaustible resources. The Review of Economic Studies, 41, 29-45. https://doi.org/10.2307/2296370.

Statistik Austria (s.a.): Wie geht's Österreich? Verfügbar in: http://www.statistik.at/web de/statistiken/wohlstand und fortschritt/wie gehts oester reich/was ist wie gehts oesterreich/index.html [Abfrage am 23.5.2019].

Steffen, W., Richardson, K., Rockström, J., Cornell, S. E., Fetzer, I., Bennett, E. M., Biggs, R., Carpenter, S. R., Vries, W. de, Wit, C. A. de, Folke, C., Gerten, D., Heinke, J., Mace, G. M., Persson, L. M., Ramanathan, V., Reyers, B., and Sörlin, S. (2015): Planetary boundaries: Guiding human development on a changing planet. Science, 347, 1259855. https://doi.org/10.1126/science.1259855.

Stiglitz, J. E., Fitoussi, J.-P., and Durand, M. (2018): Beyond GDP. Measuring What Counts for Economic and Social Performance. Paris: OECD Publishing.

Wirsenius, S., Azar, C., and Berndes, G. (2010): How much land is needed for global food production under scenarios of dietary changes and livestock productivity increases in 2030? Agricultural Systems, 103, 621-638. https://doi.org/10.1016/i.agsy.2010.07.005. 


\section{Nachhaltiges Wirtschaften \& Unternehmensmanagement}

\subsection{Unternehmerische Umwelt- und Sozialverantwortung}

Ika Darnhofer

Institut für Agrar-und Forstökonomie,

Department für Wirtschafts- und Sozialwissenschaften (WiSo)

ika.darnhofer@boku.ac.at

\subsubsection{Welche Verantwortung haben Unternehmen?}

Der Ökonom Milton Friedman beschränkte die soziale Verantwortung der Unternehmen auf die Gewinnmaximierung (Friedman 1970). Das scheint auch für die damalige Zeit etwas kurz gefasst, da Unternehmen schon immer freiwillig weitere gesellschaftliche Aufgaben übernommen haben, sei es die Förderung der Künste, die Förderung der Forschung oder auch die Bereitstellung von Wohnungen für ihre Arbeiterinnen und Arbeiter. Allerdings wird die Beschränkung der sozialen Verantwortung auf solche philanthropische Aktivitäten heute als ungenügend erachtet.

Insbesondere mit der Globalisierung, mit welcher der Einfluss von internationalen Konzernen auf die Gestaltung der Märkte und der Produktionsbedingungen gestiegen ist, wird von Unternehmen erwartet, dass sie mehr Verantwortung übernehmen. Große international agierende Konzerne beschäftigen nicht nur mehrere Hunderttausende Menschen, ihre Umsätze übertreffen häufig das Bruttoinlandsprodukt mittelgroßer Industrieländer, und sie kontrollieren zentrale Zukunftstechnologien (z.B. Gen-, Nanound Kommunikationstechnologien) (Hahn 2013). Diese multinationalen Konzerne sind politische Akteure geworden, die auf unterschiedliche Weise Einfluss auf die Gesellschaft nehmen. Entsprechend wird auch von ihnen erwartet, dass sie einen konkreten Beitrag zur nachhaltigen Entwicklung leisten.

Das Nachhaltigkeitsmanagement, d.h. die Wahrnehmung der Corporate Social Responsibility (CSR), ist zur strategischen Querschnittsaufgabe geworden. Sie umfasst die Planung, Umsetzung und Kommunikation sozialer und ökologischer Maßnahmen und deren Integration in unternehmerische Strategien. Es ist auch Teil der Organisationsentwicklung, da sich Unternehmen den sich ändernden ökologischen und gesellschaftlichen Ansprüchen stellen müssen. Nachhaltigkeitsmanagement hat daher die Aufgabe, in einer Organisation Lern- und Entwicklungsprozesse zu gestalten, damit ökologische Grenzen besser berücksichtigt werden und in der Folge das Unternehmen an der Entwicklung, wie eine gerechte Gesellschaft aussehen soll, aktiv teilnehmen kann.

Da der ökonomische Erfolg für Unternehmen dennoch wesentlich ist, stellt sich die Frage, ob die Übernahme gesellschaftlicher Verantwortung Mehrkosten verursacht 
oder ob sie dem ökonomischen Erfolg zuträglich ist. Letzteres ist der Fall, wenn z.B. eine Senkung des Material- oder Energieverbrauches Kosten spart, wenn das Image des Unternehmens verbessert wird, oder wenn die Mitarbeiterinnen und Mitarbeiter zufriedener sind und sich daher mehr engagieren. Das Argument, dass Unternehmen von der Übernahme gesellschaftlicher Verantwortung wirtschaftlich profitieren, wird als business case für CSR bezeichnet. Allerdings schränkt dieser Ansatz die Bandbreite der möglichen CSR-Aktivitäten (siehe Tabelle 2.2.1) auf jene ein, von denen ein ökonomischer Erfolg erwartet wird. Es gibt jedoch auch CSR-relevante, gesellschaftlich erwünschte Änderungen (z.B. die Eindämmung von Steuerflucht), an denen Unternehmen kein ökonomisches Interesse haben.

Tabelle 2.2.1: Beispiele für CSR-Aktivitäten (nach Meixner et al. 2015, S. 925)

\begin{tabular}{|c|c|c|c|}
\hline & Ökonomie & Ökologie & Soziales \\
\hline $\begin{array}{l}\text { Kundinnen } \\
\text { und Kunden }\end{array}$ & $\begin{array}{l}\text { - Service- und } \\
\text { Reparaturangebot }\end{array}$ & $\begin{array}{l}\text { - Kennzeichnung } \\
\text { durch Labels }\end{array}$ & $\begin{array}{l}\text { - Konsumentenschutz } \\
\text { - Information }\end{array}$ \\
\hline $\begin{array}{l}\text { Mitarbeiterinnen } \\
\text { und Mitarbeiter }\end{array}$ & $\begin{array}{l}\text { - Aufstiegsmöglichkeiten } \\
\text { - Entlohnungssystem }\end{array}$ & $\begin{array}{l}\text { - Schulung zum } \\
\text { effizienten Energie- } \\
\text { einsatz }\end{array}$ & $\begin{array}{l}\text { - Gleichbehandlung } \\
\text { - Work-Life-Balance } \\
\text { - Diversity Management }\end{array}$ \\
\hline Shareholder & $\begin{array}{l}\text { - Informationen } \\
\text { - Strategische Ziele } \\
\text { - Unternehmenswachstum }\end{array}$ & $\begin{array}{l}\text { - Ökologische } \\
\text { Kennzahlen }\end{array}$ & $\begin{array}{l}\text { - Arbeitsbedingungen als } \\
\text { Teil des CSR-Berichts }\end{array}$ \\
\hline $\begin{array}{l}\text { Gesellschaft } \\
\text { insgesamt }\end{array}$ & $\begin{array}{l}\text { Verflechtung mit } \\
\text { regionalen Unter- } \\
\text { nehmen }\end{array}$ & $\begin{array}{l}\text { - Recycling } \\
\text { - Effizienter } \\
\text { Energieeinsatz }\end{array}$ & $\begin{array}{l}\text { - Marketingethos (z.B. Ver- } \\
\text { meidung von Werbung, die } \\
\text { an Kinder gerichtet ist) } \\
\text { - Spenden und Sponsoring }\end{array}$ \\
\hline $\begin{array}{l}\text { Zuliefererinnen } \\
\text { und Zulieferer }\end{array}$ & $\begin{array}{l}\text { - Langfristige } \\
\text { Beziehungen }\end{array}$ & $\begin{array}{l}\text { - Anbau- und } \\
\text { Abbaubedingungen } \\
\text { - Saubere Produktion }\end{array}$ & $\begin{array}{l}\text { - Unterstützung zur } \\
\text { Erreichung sozialer } \\
\text { Kennzahlen }\end{array}$ \\
\hline Regierung & $\begin{array}{l}\text { - Rechtskonformität } \\
\text { - Faire Steuerleistung }\end{array}$ & $\begin{array}{l}\text { - Einhaltung gesetz- } \\
\text { licher Auflagen }\end{array}$ & $\begin{array}{l}\text { - Regelungen zum Schutz } \\
\text { der Arbeitnehmerinnen } \\
\text { und Arbeitnehmer }\end{array}$ \\
\hline
\end{tabular}

Ursprünglich kamen die Themenfelder für CSR aus dem Umweltschutz und der Einhaltung der Menschenrechte. Sie wurden schrittweise erweitert, zuerst um die Sicherheit und später um die Zufriedenheit der Mitarbeiterinnen und Mitarbeiter zu berücksichtigen. Im Folgenden wird kurz auf internationale, freiwillige Standards eingegangen, deren Bedeutung gestiegen ist, da sich internationale Konzerne zumindest teilweise dem Regelungsbereich von Nationalstaaten entziehen. Diese Standards werden von Unternehmen verwendet, um ihre Umwelt- und Sozialleistungen zu doku- 


\section{Nachhaltiges Wirtschaften \& Unternehmensmanagement}

mentieren, aber auch von Nichtregierungsorganisationen (NGOs), um Druck auf Unternehmen aufzubauen. In den folgenden Abschnitten werden exemplarisch ein paar Bereiche aus dem betrieblichen Nachhaltigkeitsmanagement angeführt, insbesondere im Bereich der sozialen Verantwortung. Für eine gesamthafte Darstellung sei auf entsprechende Lehrbücher verwiesen (z.B. Baumast und Pape 2013; Schneider und Schmidpeter 2015).

\subsubsection{Umwelt- und Sozialstandards}

Um die Aktivitäten der Unternehmen im Bereich der Nachhaltigkeit dokumentieren und vergleichen zu können, sind einheitliche Standards erforderlich. Diese können von Betrieben umgesetzt und von unabhängigen Stellen kontrolliert werden. Bei erfolgreicher Kontrolle werden Zertifikate ausgestellt, und das Unternehmen kann diese in der Kommunikation mit Stakeholdern einsetzen, z.B. indem es Labels auf Produkten anbringt. Solche Labels (z.B. Fairtrade, Forest Stewardship Council, Marine Stewardship Council, Global Organic Textile Standard) unterscheiden Produkte die ,nur' den gesetzlichen Anforderungen entsprechen, von jenen, die darüber hinausgehende Ansprüche erfüllen. Sie können wichtige Informationen für die Kaufentscheidung von Konsumentinnen und Konsumenten darstellen.

$\mathrm{Zu}$ den wichtigsten Umwelt- und Sozialstandards für Unternehmen gehören die Umweltmanagement Norm (ISO 14001) (siehe ISO 2015), das Eco-Management and Audit Scheme (EMAS) (siehe UGA 2019), die Occupational Health and Safety Management Systems (ISO 45001) (siehe ISO 2018), der Leitfaden zur gesellschaftlichen Verantwortung (ISO 26000) (siehe ISO 2010) und Social Accountability (SA8000) (siehe SAI 2014).

Standards werden laufend weiterentwickelt, u.a. um neue technische Produktionsmöglichkeiten und Änderungen in den gesellschaftlichen Erwartungen zu berücksichtigen. Auch werden stets neue Standards definiert, um relevante Prozesse differenziert bewerten und eine größere Zahl an Produkten zertifizieren zu können. Mit der Zunahme der Standards geht allerdings die Wirkung der Labels teilweise verloren, da Konsumentinnen und Konsumenten nicht immer wissen, welche ökologischen oder sozialen Standards durch ein Label vermittelt werden (siehe Beitrag 2.3).

Unternehmen sind nicht unbedingt eifrig in der Umsetzung anspruchsvoller Standards, da diese zusätzliche Auflagen im Herstellungsprozess bedeuten und die Dokumentation sowie der Zertifizierungsprozess zeitaufwendig und kostspielig sind. NGOs spielen hier eine wichtige Rolle: Sie machen auf problematische Produktionsbedingungen aufmerksam und üben damit Druck auf Unternehmen aus (siehe Fallbeispiel 2.2.1). 
Fallbeispiel 2.2.1: Initiativen für sichere und faire Arbeitsbedingungen in der Bekleidungsindustrie

In den 1990er-Jahren wurde Nike immer wieder vorgeworfen, dass die Arbeitsbedingungen, unter denen Sportartikel und -bekleidung hergestellt wurden, höchst problematisch wären, da sie u.a. mithilfe von Kinderarbeit in Pakistan und Kambodscha hergestellt wurden (Greenberg und Knight 2004). Nike vertrat ursprünglich die Position, dass das Unternehmen für die Arbeitsbedingungen in der Zulieferindustrie nicht verantwortlich sei. Der Druck der Öffentlichkeit stieg, und Anfang der 2000er-Jahre änderte Nike seine Position: Das Unternehmen gab zu, dass es hohe Anforderungen an die Qualiät seiner Produkte stellte. Warum nicht auch an die Arbeitsbedingungen? Der Sportartikelhersteller ergriff daher Maßnahmen, um bei seinen Lieferanten Kinderarbeit zu verhindern, und sorgte durch regelmäßige Berichte für Transparenz bei der Herstellung von Nike-Produkten.

Im April 2013 stürzte in Savar, Bangladesch, das achtstöckige Gebäude Rana Plaza ein, welches mehrere Textilfabriken beherbergte. Dabei kamen über 1.100 Menschen ums Leben, und mehr als 2.400 wurden verletzt, darunter mehrheitlich junge Frauen (Taplin 2014). Die Tragödie sorgte weltweit für Aufruhr und führte die Arbeitsbedingungen der Textilindustrie vor Augen. Der Bangladesh Accord (2018) wurde ins Leben gerufen, um sichere Arbeitsbedingungen für die Zukunft zu gewährleisten.

Weitere Initiativen, die sich für bessere Arbeitsbedingungen in der Textilindustrie einsetzen sind: Clean Clothes Kampagne (Südwind 2019), Sustainable Apparel Coalition (SAC 2019) und Fair Wear Foundation (FWF 2019). Eine umfassende Umsetzung dieser Initiativen würde wesentlich zur Erfüllung der Agenda 2030 für nachhaltige Entwicklung dienen, insbesondere zur Erreichung von SDG 1 keine Armut, SDG 3 Gesundheit und Wohlergehen, SDG 8 menschenwürdige Arbeit und Wirtschaftswachstum, SDG 10 weniger Ungleichheiten, sowie SDG 12 nachhaltige/r Konsum und Produktion.

\subsubsection{Betriebliche Nachhaltigkeitsberichterstattung}

Mit steigendem Bewusstsein über Missstände im Umwelt- und Sozialbereich ist auch das Informationsbedürfnis von unterschiedlichen Stakeholdern, wie Investorinnen und Investoren, Eigentümerinnen und Eigentümer, Geschäftspartnerinnen und Geschäftspartnern sowie von Konsumentinnen und Konsumenten, gestiegen. Unternehmen, die glaubwürdig über die gesellschaftlichen und ökologischen Auswirkungen ihres Handelns berichten, pflegen ihr Image - eine gute Voraussetzung für den zukünftigen Geschäftserfolg (IÖW 2008). Dieser Vertrauensaufbau gelingt besonders dann, wenn die regelmäßige Berichterstattung Änderungen und stetige Weiterentwicklung dokumentiert. So kann ein Unternehmen überzeugend vermitteln, dass es seiner gesellschaftlichen Verantwortung gerecht wird.

Ziel der Berichte ist es, transparent Rechenschaft über die Leistungen eines Unternehmens zu legen und darüber zu berichten, welche innovativen und nachhaltigen Antworten es auf wichtige aktuelle Herausforderungen gefunden hat. Um Qualitätsanforderungen an CSR-Berichten zu definieren und die Vergleichbarkeit der Leistungskennzahlen zu Nachhaltigkeitsthemen sicherzustellen, gibt es Richtlinien wie die AA1000 Prinzipien (AccountAbility 2008) bzw. die GRI-Standards (GRI 2018). 


\section{Nachhaltiges Wirtschaften \& Unternehmensmanagement}

Die GRI-Standards geben detaillierte Hinweise zu Datenerhebung, Systemgrenzen, Berichterstellung und branchenspezifische Empfehlungen.

Auch die Europäische Kommission hat eine Richtlinie für die Angabe nichtfinanzieller und die Diversität betreffender Informationen durch große Unternehmen erlassen (NFI-Richtlinie 2014). Seit Jänner 2018 haben alle Unternehmen mit mehr als 500 Mitarbeiterinnen und Mitarbeitern jährlich nichtfinanzielle Informationen zu veröffentlichen. Diese Berichte haben folgende Themen zu umfassen: Umweltschutz, soziale Verantwortung und Belange der Arbeitnehmerinnen und Arbeitnehmer, Achtung der Menschenrechte, Bekämpfung von Korruption und Bestechung sowie ein Diversitätskonzept im Zusammenhang mit den Verwaltungs-, Leitungs- und Aufsichtsorganen des Unternehmens. Bemerkenswert dabei ist, dass die Europäische Kommission die Nachhaltigkeitsberichterstattung nicht mehr als freiwillig erachtet. Es wird erwartet, dass Unternehmen ihre soziale Verantwortung wahrnehmen und transparent darüber berichten.

Die NFI-Richtlinie (2014) wurde in Österreich in Form des Nachhaltigkeits- und Diversitätsverbesserungsgesetzes ( $\mathrm{NaDiVeG} 2016)$ umgesetzt. Knapp 120 große Unternehmen von öffentlichem Interesse sind nun verpflichtet, wesentliche Informationen zur Nachhaltigkeitsleistung in Form einer Nichtfinanziellen Erklärung (NFI-Erklärung) im Lagebericht oder in einem gesonderten Nachhaltigkeitsbericht zu veröffentlichen. Neben dieser gesetzlichen Verpflichtung für große Betriebe zeichnen sich in Österreich viele Klein- und Mittelbetriebe durch sehr engagierte Nachhaltigkeitsberichte aus (Jasch 2015, S. 830).

\subsubsection{Nachhaltiges Management von Wertschöpfungsketten}

Der alleinige Fokus auf Umwelt- und Sozialaspekte in den eigenen Produktionsstätten reicht häufig nicht mehr. Dies liegt daran, dass Leistungen kaum noch von einem Unternehmen allein erbracht werden: Es sind unterschiedliche Lieferantinnen und Lieferanten in den Leistungserstellungsprozess eingebunden. In manchen Branchen, wie z.B. in der Automobilindustrie oder im Einzelhandel, werden mehr als 60\% der Wertschöpfung in vorgelagerten Unternehmen erbracht (Seuring und Müller 2013, S. 245).

Um dieser Produktionsstruktur gerecht zu werden, nimmt die Bedeutung vom ,Supply Chain Management ${ }^{`}$ zu. Es zielt einerseits darauf ab, Material-, Produktions- und Informationsflüsse zu optimieren, und andererseits Kooperationen mit Zulieferbetrieben, mit NGOs sowie mit Kundinnen und Kunden proaktiv zu gestalten. Ziel ist es, die gesamte Wertschöpfungskette als eine Einheit zu betrachten, da der Wettbewerb nicht mehr nur zwischen Unternehmen, sondern auch zwischen Wertschöpfungsketten stattfindet.

Diese Wertschöpfungsketten sind heute überwiegend global, und häufig wird in sogenannten ,Billiglohnländern' produziert. Allerdings herrschen in diesen Ländern für 
westliche Konsumentinnen und Konsumenten oftmals inakzeptable Umwelt- und Arbeitsbedingungen (siehe Fallbeispiel 2.2.1). ${ }^{1}$ Auf Umwelt- und Sozialthemen fokussierte NGOs berichten regelmäßig über Missstände auf den Vorstufen der Wertschöpfungskette. Großangelegte Kampagnen können erheblichen Druck auf Unternehmen ausüben. Bekannte Beispiele sind Kampagnen, die auf die Arbeitsbedingungen in chinesischen Unternehmen, in denen Apple-Produkte hergestellt werden (Frost und Burnett 2007), oder den Umgang von Starbucks mit Kaffeebäuerinnen und -bauern (Argenti 2004) aufmerksam gemacht haben.

Da die Reputation der Marke durch solche Kampagnen gefährdet wird, sind die Unternehmen bestrebt, in ihren Beziehungen zu Lieferantinnen und Lieferanten nicht nur ökonomische, sondern auch soziale und ökologische Aspekte zu berücksichtigen. Dabei arbeiten zukunftsweisende Unternehmen mit unterschiedlichen Stakeholdern zusammen, um Ziele zu definieren und Veränderungsprozesse mit den Lieferantinnen und Lieferanten zu gestalten (Frost und Burnett 2007). Ein qualitativ gutes Engagement der Stakeholder kann zu einer gerechteren gesellschaftlichen Entwicklung führen, indem denjenigen, die das Recht haben, gehört zu werden, in den Entscheidungsfindungsprozess einbezogen werden. Der Stakeholder Engagement Standard (AA1000SES) (siehe AccountAbility 2015) definiert einen Rahmen für die Bewertung, Gestaltung, Umsetzung und Kommunikation eines hochwertigen Austauschprozesses mit unterschiedlichen Anspruchs- und Interessensgruppen.

\subsubsection{Diversity Management}

Die soziale Verantwortung von Unternehmen betrifft auch ganz wesentlich den Umgang mit den eigenen Mitarbeiterinnen und Mitarbeitern. Hier spielt Diversity Management eine immer größere Rolle. Darunter fallen Maßnahmen zur Frauenförderung (Gender Pay Gap reduzieren; Frauenanteil in Führungspositionen erhöhen), Work-Life-Balance, die bessere Einbindung ethnischer Minderheiten oder auch altersgerechte Arbeitsplatzgestaltung. Damit spiegelt Diversity die zahlreichen Veränderungen in der heutigen Gesellschaft wider, wie z.B. die Alterung der Bevölkerung, das neue Selbstverständnis der Frauen in der Arbeitswelt oder die ethnische Vielfalt.

Ziel eines effektiven Diversity Managements ist es, Unterschiede als strategische Ressource zu nutzen, d.h. Ausgrenzung zu vermeiden und die personelle Vielfalt als Bereicherung wahrzunehmen. Eine Unternehmenskultur, die Diversität wertschätzt, ermöglicht es den Mitarbeiterinnen und Mitarbeitern, ihre einzigartigen Fähigkeiten zu entfalten, und erhöht ihre Motivation; damit können Fehlzeiten und Personal-

1 In vielen Ländern gibt es Vorschriften zu sozialen Fragen, wie z.B. Mindestlohn, Kinderarbeit oder Arbeitszeiten, sowie zum Umweltschutz. Allerdings besteht ein erhebliches Vollzugsdefizit, da Behörden nicht immer in der Lage sind, diese Vorschriften zu kontrollieren und wirkungsvoll einzufordern. 


\section{Nachhaltiges Wirtschaften \& Unternehmensmanagement}

fluktuationen reduziert werden (Hanappi-Egger 2015). Dazu müssen unterschiedliche Dimensionen von Vielfalt bei Mitarbeiterinnen und Mitarbeitern betrachtet werden: die Persönlichkeit, innere Dimensionen (z.B. Geschlecht, Alter, sexuelle Orientierung, ethnische Zugehörigkeit), äußere Dimensionen (Familienstand, Ausbildung, Einkommen) und organisationale Dimensionen (Dauer der Zugehörigkeit, Arbeitsfeld, Funktion) (Gardenschwarz und Rowe 2003). Diversity Management bedeutet daher eine differenziertere Personalpolitik, ein Hinterfragen von Homogenität, eine Abkehr der Orientierung am stereotypen ,Norm-Mitarbeiter ' (dem weißen, heterosexuellen, verheirateten Mann).

Auch im Umgang mit Kundinnen und Kunden kann Diversity Management eine Rolle spielen, indem z.B. bei der Entwicklung von Produkten oder Dienstleistungen die Bedürfnisse spezieller Altersgruppen oder Ethnien berücksichtigt und diese Gruppen gezielt angesprochen werden (z.B. Ethno-Marketing).

\subsubsection{Unternehmensethik}

Unternehmensethik umfasst Compliance (d.h. Regeleinhaltung) und Integrität (d.h. die Fähigkeit und Bereitschaft zu eigenverantwortlichem Handeln in schwierigen Situationen) (Clausen 2009, S. 32). Beide sind gleichermaßen wichtig: Regeln geben Orientierung für das eigene Handeln. Sie können aber nie alle Situationen erfassen. Daher ist die intrinsische Motivation zu richtigem Verhalten wesentlich.

Dass ethisches Verhalten nicht vorausgesetzt werden kann, zeigt sich daran, dass namhafte Unternehmen wiederholt aufgrund ihrer Geschäftspraktiken mit negativen Schlagzeilen in die Medien kommen. Diese Praktiken sind teilweise illegal und teilweise innerhalb des gesetzlichen Rahmens, aber jedenfalls unethisch. So verwenden internationale Konzerne wie Starbucks, Fiat und Amazon kreative Mechanismen und Gesellschaftskonstruktionen, um ihre Steuerschuld zu reduzieren (siehe Fallbeispiel 2.2.2).

\section{Fallbeispiel 2.2.2: Steuervermeidung durch Starbucks (EU COM 2015a)}

Der Starbucks-Konzern hat ein kompliziertes Geflecht an Tochtergesellschaften in verschiedenen europäischen Ländern aufgebaut und nutzt es, um Geldflüsse steuermindernd zu gestalten. So betreibt Starbucks in den Niederlanden eine Kaffeerösterei und produziert dort auch Becher, Servietten und andere Utensilien, die in alle Filialen in Europa exportiert werden. Für die Verwendung von Knowhow zahlt diese Kaffeerösterei Lizenzgebühren an die Tochtergesellschaften und reduziert dadurch den steuerbaren Gewinn in den Niederlanden. Der Gewinn wird noch weiter reduziert, indem sie die zur Röstung vorgesehenen Bohnen zu einem stark überhöhten Preis von einem anderen Tochterunternehmen der Starbucks-Gruppe in der Schweiz erwirbt. Auch die Starbucks-Tochter in Österreich zahlt eine Lizenzgebühr an die Zentrale in den Niederlanden, wodurch die Steuerleistung in Österreich reduziert wird. 
Dies wirkt nicht nur wettbewerbsverzerrend, sondern schadet auch Nationalstaaten durch geringere Steuereinnahmen. Die Europäische Kommission hat daher eine Initiative zur ,fairen Besteuerung' (EU COM 2018) gestartet, ein ,Steuertransparenz-

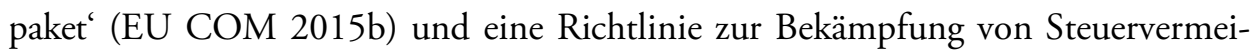
dungspraktiken (Anti-BEPS-Richtlinie 2016) verabschiedet, deren Vorschriften mit 1. Jänner 2019 anzuwenden sind.

Beispiele für ethisch bedenkliche und illegale Geschäftspraktiken liefert der Volkswagen-Konzern (siehe Fallbeispiel 2.2.3). Eine wichtige Frage in diesem Kontext ist, wie es zu solchen Vorgängen kommen kann, in die viele Personen involviert sind und die sich über längere Zeiträume erstrecken. Offensichtlich spielen dabei nicht nur individuelle Handlungsmotive (u.a. Streben nach Macht und Einfluss, persönliche Bereicherung, mangelndes Unrechtsbewusstsein) eine Rolle, sondern auch Organisationsstrukturen. Diese können zu Rollenkonflikten und zu einer Unternehmenskultur von gegenseitigen Gefälligkeiten führen - eine Voraussetzung für Missstände, wie sie in den Fallbeispielen angeführt wurden (Clausen 2009).

\section{Fallbeispiel 2.2.3: Unethische und illegale Praktiken beim VW-Konzern (Clausen 2009)}

Im Jahr 2005 gerät der Volkswagen Konzern mit Meldungen über Lustreisen für Betriebsratsangehörige in die Schlagzeilen: WW zahlt Dienstleistungen von Prostituierten, Geschenke für Ehefrauen und Honorare sowie Reisekosten in Höhe von mehr als 1 Mio. $€$ allein für die Geliebte des Gesamtbetriebsratsvorsitzenden Klaus Volkert. Sich auf Firmenkosten zu vergnügen, ist Untreue (Verwendung von Vermögensgütern entgegen der erteilten Verfügungsbefugnis) und Betrug (Verfügung über das Vermögen der/des Getäuschten, ohne angemessene Gegenleistung). Des Weiteren kassierte Volkert selbst Prämienzahlungen in Millionenhöhe, was gegen das Betriebsverfassungsgesetz verstößt. Die Vorgänge haben Konsequenzen: Volkert (und andere) treten im Juni 2005 zurück (Clausen 2009, S. 15ff.).

Die deutsche Automobilindustrie ist dafür bekannt, dass sie politische Lobbyarbeit leistet, um die Verschärfung von Umweltnormen zu verzögern oder abzumildern. Dass der VW-Konzern wenig sensibel für gesellschaftliche Anliegen wie Klimaschutz ist, zeigte sich auch im Jahr 2015, als die Manipulation von Dieselmotoren und der Einbau von Betrugssoftware öffentlich wurde. Damit wird deutlich, dass WW Abgasvorschriften nicht als Mindestnorm sieht, geschweige denn versucht, diese zu übertreffen.

Diese Beispiele zeigen, dass sich Unternehmen auch in Europa nicht immer redlich verhalten und sich Spitzenmanagerinnen und -manager in ethische, moralische oder juristische Graubereiche begeben. Eine Studie von Ernst \& Young (2017) zeigt, dass $23 \%$ der befragten deutschen Führungskräfte für das eigene berufliche Fortkommen zumindest zu einer der folgenden Verhaltensweisen bereit wären: Externe täuschen, das eigene Management mit falschen Informationen versorgen und unethisches Verhalten bei Kundinnen und Kunden, Lieferantinnen und Lieferanten oder im eigenen Team ignorieren. 


\section{Nachhaltiges Wirtschaften \& Unternehmensmanagement}

Eine wesentliche Aufgabe von Unternehmensethik ist, die Urteilsfähigkeit von Entscheidungsträgerinnen und Entscheidungsträgern zu stärken, damit sie die Fähigkeit aufbauen, sich eine fundierte Meinung über eine Situation zu bilden und deren ethische Dimension zu erkennen. Nur dann ist es möglich, vermeintlichen Sachzwängen zu entkommen, die vorhandenen Handlungsmöglichkeiten zu erkennen und kreativ zu nutzen. Wesentlich ist daher die Fähigkeit, die derzeitigen Spielregeln zu reflektieren und sich immer wieder die Kernfrage der Unternehmensethik zu stellen: Wie wollen wir im Wirtschaftsleben miteinander umgehen (Clausen 2009)?

\subsubsection{Nächste Schritte: Suffizienz, Social Entrepreneurship}

Viele Unternehmen verfolgen einen complianceorientierten Ansatz zur unternehmerischen Verantwortung und beschränken sich darauf, Gesetze und Branchenstandards einzuhalten sowie ihre Mitarbeiterinnen und Mitarbeiter fair zu behandeln. Sie fokussieren sich daher primär auf Risikomanagement, indem sie auf ihr Image und das Medienecho achten und die Interessen der lautesten Stakeholder befriedigen. Manche Unternehmen gehen weiter und sehen ihre gesellschaftliche Verantwortung darin, zu einem drängenden gesellschaftlichen Problem einen Lösungsbeitrag zu leisten.

So wählen manche Suffizienz als Unternehmensstrategie. Indem sie etablierte Produktions- und Konsummuster hinterfragen, leisten sie einen Beitrag zu den Zielen der Agenda 2030, insbesondere zu SDG 12 für nachhaltige Konsum- und Produktionsmuster. Konkrete Beispiele für die Umsetzung von Suffizienzstrategien sind z.B.: den Schwerpunkt auf einfache und langlebige Produkte zu legen; Entschleunigung zu unterstützen, indem z.B. Telefondienstleister Kundinnen und Kunden einen Rabatt anbieten, wenn sie nicht jährlich das Mobiltelefon wechseln; eine Entkommerzialisierung z.B. durch Bildungsangebote, die Fähigkeiten vermitteln, Leistungen selbst erbringen zu können; oder eine konsequente Orientierung an regionalen Zulieferbetrieben, um Transportwege zu reduzieren (Baumast 2013). Als Beispiel für die Umsetzung einer Suffizienzstrategie kann auch die ,Common Threads Initiative' des Outdoorbekleidungsherstellers Patagonia (2011a) dienen: Durch Anzeigen rief Patagonia (2011b) dazu auf, eine Jacke nicht zu kaufen, außer man brauche sie wirklich. Patagonia lud damit ein, sich an den Prinzipien Reduzieren, Reparieren, Weiterverwenden, Recyclen und Umdenken zu orientieren (zum Beitrag der Konsumentinnen und Konsumenten zu Suffizienz siehe Beitrag 2.3).

Noch weiter gehen Social Entrepreneurs, die gesellschaftliche Interessen als wesentliche Grundlage ihrer Unternehmensstrategie sehen. Bei der Gestaltung ihrer Geschäftstätigkeit orientieren sie sich daher an fundamental anderen Fragen: Fördert 
der Geschäftszweck eine nachhaltige Entwicklung? Welche Konsummuster werden initiiert? Welche Lebensstile werden unterstützt? Mit anderen Worten: Was bewirkt die unternehmerische Tätigkeit (Schaltegger 2017)?

Social Entrepreneurs wollen auf diese Weise aktiv und kreativ zur Transformation unseres Wirtschaftssystems beitragen. Sie stellen sich der Herausforderung, neue Unternehmensmodelle zu entwickeln, welche die Ressourcen der nachfolgenden Generationen nicht nur erhalten, sondern vermehren und somit zukünftige Handlungsspielräume unserer Gesellschaft vergrößern. Die bisherigen - oftmals nur auf kurzfristigen Gewinn ausgerichteten - Geschäftsmodelle werden völlig neu gedacht, sodass die Wertschöpfung für Gesellschaft und Unternehmen das übergeordnete Ziel werden (Schmidpeter 2015). Social Entrepreneurs verfolgen daher keine primäre oder ausschließliche Profitorientierung. In der Regel zielen sie auf Kostendeckung $a b$ und reinvestieren die Gewinne im Sinne des Gründungsziels.

Social Entrepreneurs sind häufig im Kontext der Entwicklungszusammenarbeit tätig. Sie entwickeln z.B. technische Lösungen für eine kostengünstige Produktion von sauberem Strom oder Trinkwasser für arme Bevölkerungsgruppen. Als eines der bekanntesten Beispiele gilt der Nobelpreisträger Muhammad Yunus, der Gründer der Grameen Bank (2019), die Mikrokredite an gesellschaftliche Randgruppen vergibt. Diesen Randgruppen wurden dadurch neue Möglichkeiten eröffnet, da sie bisher vom Finanzsystem ausgeschlossen waren.

\subsubsection{Ausblick: ein lohnendes, breites Tätigkeitsfeld}

Unternehmen produzieren Waren oder Dienstleistungen und sind grundsätzlich auf finanziellen Gewinn ausgerichtet. Herkömmliche Unternehmen orientieren sich an der Gewinnmaximierung bzw. Verlustminimierung; sie nehmen gesellschaftliche Erwartungen primär über die Gesetze und die Nachfrage von Kundinnen und Kunden wahr. In den letzten Jahrzehnten ist der Druck auf Unternehmen gestiegen, über diese Mindestanforderungen hinaus einen Beitrag zur nachhaltigen Entwicklung zu leisten. Dieser fällt ganz unterschiedlich aus, je nachdem, welche Probleme in einer Branche oder Region besonders akut sind (Abbildung 2.2.1). Ursprünglich war es v.a. die Erwartung, dass Unternehmen freiwillig mehr für den Umweltschutz tun, später ist der Umgang mit Mitarbeiterinnen und Mitarbeitern stärker ins Blicklicht gerückt. Immer mehr Unternehmen wollen unmittelbar durch ihre Aktivitäten einen positiven Wandel in der Gesellschaft herbeiführen. Im Mittelpunkt der Unternehmensethik steht für sie weniger die Gewinnmaximierung, sondern einen Beitrag zur Lösung von gesellschaftlichen Problemen zu leisten und dabei Gewinn zu erwirtschaften. 


\section{Nachhaltiges Wirtschaften \& Unternehmensmanagement}

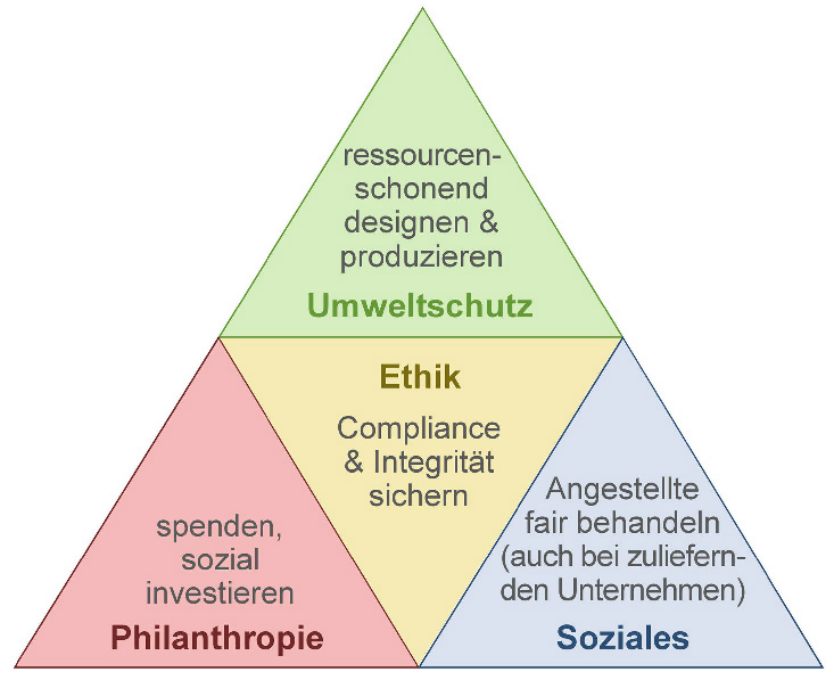

Abbildung 2.2.1: Die unterschiedlichen Bereiche der unternehmerischen Umweltund Sozialverantwortung

Betriebliches Nachhaltigkeitsmanagement bietet damit ein breites Tätigkeitsfeld für UBRM-Absolventinnen und -Absolventen. Dabei geht es nicht nur um die Gestaltung von technischen Prozessen oder Umweltschutz, sondern auch um soziale Verantwortung. Im Vordergrund stehen wissenschaftlich fundierte Erkenntnisse und die Fähigkeit, ethisch begründete Richtungsentscheidungen treffen zu können. Nachhaltigkeitsmanagerinnen und -manager können Initiativen partnerschaftlich mit Stakeholdern ausverhandeln, die Unternehmensleitung von deren strategischer Bedeutung überzeugen und sie wirkungsvoll an Kundinnen und Kunden kommunizieren. Indem sie Möglichkeiten aufzeigen, wie Unternehmen dem Ressourcenverbrauch, Umweltschäden und sozialen Missständen entgegenwirken können, werden sie ihrer gesellschaftlichen Verantwortung gerecht und legen wichtige Grundbausteine für eine nachhaltige Zukunft.

\section{Literatur}

AccountAbility (2008): AA1000 Accountability Prinzipien Standard 2008. London, UK. Verfügbar in: https://www.accountability.org/standards/ [Abfrage am 20.7.2019].

AccountAbility (2015): AA1000 Stakeholder Engagement Standard 2015. London, UK. Verfügbar in: https://www.accountability.org/standards/ [Abfrage am 20.7.2019].

Anti-BEPS-Richtlinie (2016): Richtlinie (EU) 2016/1164 des Rates vom 12. Juli 2016 mit Vorschriften zur Bekämpfung von Steuervermeidungspraktiken mit unmittelbaren Auswirkungen auf das Funktionieren des Binnenmarkts. Abl L 193/2016, 1. Verfügbar in: http://data.europa.eu/eli/dir/2016/1164/oj [Abfrage am 20.7.2019].

Argenti, P. (2004): Collaborating with activists: How Starbucks works with NGOs. California Management Review, 47, 1, 91-116. https://doi.org/10.2307/41166288. 
Bangladesh Accord (Accord on Fire and Building Safety in Bangladesh) (2018): Website of the Bangladesh Accord. Available at: https://bangladeshaccord.org/ [accessed 20.7.2019].

Baumast, A. (2013): Perspektive Nachhaltigkeit - Effizienz, Konsistenz und Suffizienz als Unternehmensstrategien. In: Baumast, A. und Pape, J., Hrsg., Betriebliches Nachhaltigkeitsmanagement. Stuttgart: Eugen Ulmer, 360-373.

Baumast, A. und Pape, J. (Hrsg.) (2013): Betriebliches Nachhaltigkeitsmanagement. Stuttgart: Eugen Ulmer.

Clausen, A. (2009): Grundwissen Unternehmensethik. Tübingen: A. Franke UTB.

Ernst \& Young (2017): EMEIA Fraud Survey - Ergebnisse für Deutschland, April 2017. Düsseldorf. Verfügbar in: https://www.ey.com/ [Abfrage am 17.3.2019].

EU COM (Europäische Kommission) (2015a): Kommission stellt Unvereinbarkeit der selektiven Steuervorteile für Fiat in Luxemburg und für Starbucks in den Niederlanden mit dem EUBeihilfenrecht fest. Pressemitteilung. Verfügbar in: http://europa.eu/rapid/press-release IP15-5880 de.htm [Abfrage am 17.3.1019].

EU COM (Europäische Kommission) (2015b): Maßnahmenpaket zur Steuertransparenz. Verfügbar in: https://ec.europa.eu/taxation customs/business/company-tax/tax-transparencypackage de [Abfrage am 20.7.2019].

EU COM (European Commission) (2018): Proposal for a Council Directive laying down rules relating to the corporate taxation of a significant digital presence. $\operatorname{COM(2018)~} 147$ final. Available at: https://ec.europa.eu/taxation customs/business/company-tax/fair-taxationdigital-economy en [accessed 20.7.2019].

Friedman, M. (1970): The social responsibility of business is to increase its profits. The New York Times Magazine, 13 Sept. 1970. Available at: http://umich.edu/ thecore/doc/Friedman.pdf [accessed 17.3.2019].

Frost, S. and Burnett, M. (2007): Case study: The Apple iPod in China. Corporate Social Responsibility and Environmental Management, 14, 103-113. https://doi.org/10.1002/csr.146.

FWF (Fair Wear Foundation) (2019): Website of the FWF. Amsterdam, the Netherlands. Available at: https://www.fairwear.org/ [accessed 20.7.2019].

Gardenschwartz, L. and Rowe, A. (2003): Diverse Teams at Work. Capitalizing on the Power of Diversity. Alexandria, VA, USA: Society for Human Resource Management.

Grameen Bank (2019): Website of the Grameen Bank. Dhaka, Bangladesh. Available at: http://www.grameen.com/introduction/ [accessed 20.7.2019].

Greenberg, J. and Knight, G. (2004): Framing sweatshops: Nike, global production and the American news media. Communication and Critical/Cultural Studies, 1, 151-175. https://doi.org/10.1080/14791420410001685368.

GRI (Global Reporting Initiative) (2018): GRI Sustainability Reporting Standards. Global Sustainability Standards Board (GSSB), Amsterdam, the Netherlands. Available at: https://www.globalreporting.org/standards/gri-standards-translations/ [accessed 20.7.2019].

Hahn, R. (2013): Corporate Citizenship - Unternehmen als politische Akteure. In: Baumast, A., und Pape, J., Hrsg., Betriebliches Nachhaltigkeitsmanagement. Stuttgart: Eugen Ulmer, 123-138.

Hanappi-Egger, E. (2015): Diversitätsmanagement und CSR. In: Schneider, A. und Schmidpeter, R., Hrsg., Corporate Social Responsibility. 2. Auflage. Berlin: Springer-Gabler, 211-223.

IÖW (Institut für Ökologische Wirtschaftsforschung) (Hrsg.) (2008): Leitlinie zu wesentlichen nichtfinanziellen Leistungsindikatoren, insbesondere zu Umwelt- und ArbeitnehmerInnenbelangen, im Lagebericht. Wien (Brom, M., Frey, B., Jasch, C., Projektpartnerinnen). Verfügbar in: http://www.ioew.at/ioew/d-ioew-set.html [Abfrage am 20.7.2019].

ISO (International Organization for Standardization) (2010): ISO 26000:2010 Guidance on Social Responsibility. Geneva, Switzerland. Available at: https://www.iso.org/iso-26000-socialresponsibility.html [accessed 20.7.2019].

ISO (International Organization for Standardization) (2015): ISO 14001:2015 Environmental Management Systems - Requirements with Guidance for Use. Geneva, Switzerland. Available at: https://www.iso.org/standard/60857.html [accessed 20.7.2019]. 


\section{Nachhaltiges Wirtschaften \& Unternehmensmanagement}

ISO (International Organization for Standardization) (2018): ISO 45001:2018 Occupational Health and Safety Management Systems - Requirements with Guidance for Use. Geneva, Switzerland. Available at: https://www.iso.org/iso-45001-occupational-health-and-safety.html [accessed 20.7.2019].

Jasch, C. (2015): CSR und Berichterstattung. In: Schneider, A. und Schmidpeter, R., Hrsg., Corporate Social Responsibility. 2. Auflage. Berlin: Springer-Gabler, 823-834.

Meixner, O., Schwarzbauer, A. und Pöchtrager, S. (2015): CSR in der Agrar- und Ernährungswirtschaft. In: Schneider, A. und Schmidpeter, R., Hrsg., Corporate Social Responsibility. 2. Auflage. Berlin: Springer-Gabler, 921-932.

NaDiVeG (2016): Bundesgesetz, mit dem zur Verbesserung der Nachhaltigkeits- und Diversitätsberichterstattung das Unternehmensgesetzbuch, das Aktiengesetz und das GmbH-Gesetz geändert werden (Nachhaltigkeits- und Diversitätsverbesserungsgesetz, NaDiVeG). BGBI. I Nr. 20/2017. Verfügbar in: https://www.ris.bka.gv.at/eli/bgbl/I/2017/20/20170117 [Abfrage am 20.7.2019].

NFI-Richtlinie (2014): Richtlinie 2014/95/EU des Europäischen Parlaments und des Rates vom 22. Oktober 2014 zur Änderung der Richtlinie 2013/34/EU im Hinblick auf die Angabe nichtfinanzieller und die Diversität betreffender Informationen durch bestimmte große Unternehmen und Gruppen. Abl L 330/2014, 1. Verfügbar in: http://data.europa.eu/eli/dir/2014/95/oj [Abfrage am 20.7.2019].

Patagonia (2011a): Common Threads Initiative. Patagonia blog, Sept. 2011. Ventura, CA, USA. Available at: https://www.patagonia.com/blog/2011/09/introducing-the-commonthreads-initiative/ [accessed 20.7.2019].

Patagonia (2011b): Don't buy this jacket. Patagonia advertisement on Black Friday, New York Times, Nov. 25, 2011. Available at: https://www.patagonia.com/blog/2011/11/dont-buy-thisjacket-black-friday-and-the-new-york-times/ [accessed 20.7.2019].

SAC (Sustainable Apparel Coalition) (2019): Website of the SAC. San Francisco, CA, USA. Available at: https://apparelcoalition.org [accessed 20.7.2019].

SAI (Social Accountability International) (2014): SA8000 ${ }^{\circledR}$ Standard. New York, USA. Available at: http://www.sa-intl.org [accessed 20.7.2019].

Schaltegger, S. (2017): Sustainable Entrepreneurship als Treiber von Transformation. Verfügbar in: https://www.zukunftsinstitut.de/artikel/sustainable-entrepreneurship-als-treiber-vontransformation/ [Abfrage am 17.3.2019].

Schmidpeter, R (2015): CSR, Sustainable Entrepreneurship und Social Innovation - Neue Ansätze der Betriebswirtschaftslehre. In: Schneider, A. und Schmidpeter, R., Hrsg., Corporate Social Responsibility. 2. Auflage. Berlin: Springer-Gabler, 135-144.

Schneider, A. und Schmidpeter, R. (Hrsg.) (2015): Corporate Social Responsibility. Verantwortungsvolle Unternehmensführung in Theorie und Praxis. 2. Auflage. Berlin: Springer-Gabler.

Seuring, S. und Müller, M. (2013): Nachhaltiges Management von Wertschöpfungsketten. In: Baumast, A und Pape, J., Hrsg., Betriebliches Nachhaltigkeitsmanagement. Stuttgart: Eugen Ulmer, 245-258.

Südwind (Südwind - Verein für Entwicklungspolitik und globale Gerechtigkeit) (2019): Webseite der Clean Clothes Kampagne Österreich. Wien. Verfügbar in: https://www.cleanclothes.at/ [Abfrage am 20.7.2019].

Taplin, I. (2014): Who is to blame? A re-examination of fast fashion after the 2013 factory disaster in Bangladesh. Critical Perspectives on International Business, 10, 1/2, 72-83. https://doi.org/10.1108/cpoib-09-2013-0035.

UGA (Umweltgutachterausschuss beim Bundesministerium für Umwelt, Naturschutz und nukleare Sicherheit) (2019): Webseite des Eco-Management and Audit Scheme (EMAS). Berlin. Verfügbar in: https://www.emas.de/ [Abfrage am 20.7.2019]. 


\subsection{Nachhaltiger Konsum}

Petra Riefler und Laura Wallnöfer

Institut für Marketing und Innovation,

Department für Wirtschafts- und Sozialwissenschaften (WiSo)

petra.riefler@boku.ac.at, laura.wallnoefer@boku.ac.at

\subsubsection{Die Rolle von Konsumentinnen und Konsumenten für eine nachhaltige Wirtschaft}

„Essen Sie weniger Fleisch - der Umwelt zuliebe!“ Mit solchen und ähnlichen Appellen richten sich Natur- und Umweltschutzorganisationen an Konsumentinnen und Konsumenten. Sie dienen v.a. der Bewusstseinsbildung in der Bevölkerung über den direkten Zusammenhang des eigenen Konsums und dem Klimawandel (siehe Fallbeispiel 2.3.1 und Abbildung 2.3.1). Konsumentinnen und Konsumenten treffen

Fallbeispiel 2.3.1: Nachhaltiger Lebensmittelkonsum, WWF Warenkorbstudie (2019)

Der wöchentliche Lebensmitteleinkauf einer durchschnittlichen österreichischen vierköpfigen Familie verursacht rund $58 \mathrm{~kg}$ an $\mathrm{CO}_{2}$-Emissionen (vgl. WWF 2019). Würde sich diese durchschnittliche Familie für mehr Hülsenfrüchte, Obst und Gemüse aus biologischem Anbau und für weniger Fleischwaren entscheiden, verringerten sich ernährungsbedingte Treibhausgasemissionen um bis zu $40 \%$.

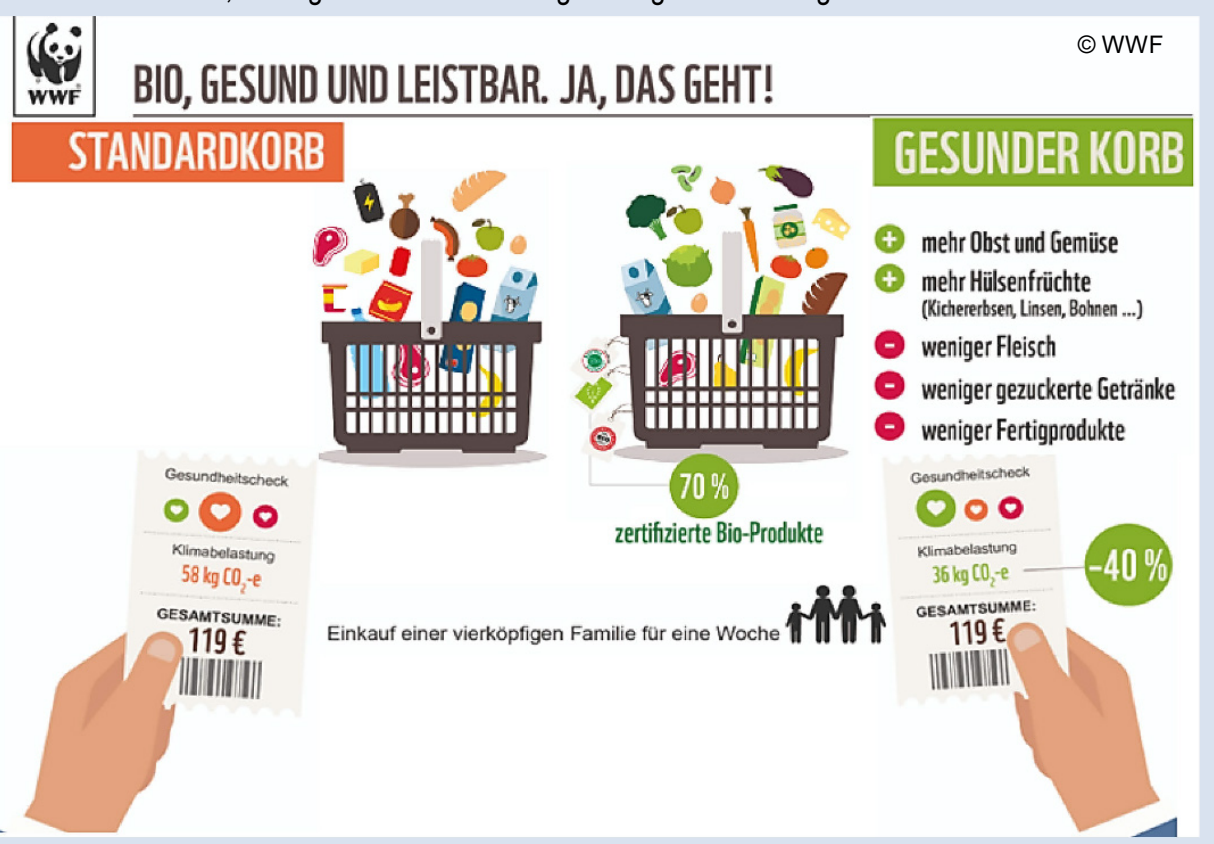

Abbildung 2.3.1: Vergleich eines üblichen Warenkorbs (links) mit einem nachhaltigeren und gesünderen Warenkorb (rechts) (WWF 2019) 


\section{Nachhaltiges Wirtschaften \& Unternehmensmanagement}

täglich eine Vielzahl an Entscheidungen, die - für sie häufig unsichtbare - soziale und ökologische Konsequenzen haben.

Schätzungen zufolge gehen zwischen 50 und $80 \%$ des weltweiten Land-, Materialund Wasserverbrauchs sowie mehr als $60 \%$ der konsumbasierten Treibhausgasemissionen auf die Nachfrage privater Haushalte zurück. Die relevantesten Konsumbereiche sind in diesem Zusammenhang Ernährung, individuelle Mobilität und Wohnen (vgl. Hertwich et al. 2015). Wie diese Zahlen verdeutlichen, spielen Konsumentinnen und Konsumenten - neben Regierungen, Unternehmen sowie anderen Akteurinnen und Akteuren - eine zentrale Rolle bei der Gestaltung eines nachhaltigen Wirtschaftssystems (vgl. UN 2016).

Das SDG 12 (nachhaltige Konsum- und Produktionsmuster) greift diese zentrale Rolle auf. Während Beitrag 2.2 die unternehmerische Verantwortung zur Erreichung der Ziele des SDG 12 beleuchtet hat, beschäftigt sich dieser Beitrag mit der Verantwortung von Konsumentinnen und Konsumenten. Zum einen sind Corporate-SocialResponsibility-Aktivitäten von Unternehmen, die im Beitrag 2.2 beschrieben wurden, in vielen Fällen nur dann erfolgreich, wenn Konsumentinnen und Konsumenten bereit sind, Unternehmen zu wählen, die gesellschaftliche Verantwortung übernehmen (Riefler 2019). Zum anderen besteht breiter Konsens darüber, dass Konsumentinnen und Konsumenten eigene Konsummuster insgesamt nachhaltiger gestalten müssten, um die Ziele des SDG 12 zu erreichen.

Um Veränderungen im Sinne des SDG 12 zu erreichen, ist es folglich entscheidend, individuelle Werte, Gewohnheiten und Entscheidungsmuster grundlegend zu kennen und bestimmende Einflussfaktoren (z.B. aus dem kulturellen und sozialen Umfeld) auf das Konsumverhalten zu verstehen. Dieses Verständnis ermöglicht es, nachhaltige Innovationen und Geschäftsmodelle so zu gestalten, dass sie individuellen Bedürfnissen bestmöglich entsprechen. Dieses Verständnis ist auch die Basis für die Ausgestaltung von Anreizen für individuelle Verhaltensänderungen im Sinne eines umweltund sozialverträglichen Konsums. Die Konsumentenforschung trägt dazu bei, Antworten auf Fragen wie jene am Beginn dieses Beitrages zu finden: Wie kann man mehr Konsumentinnen und Konsumenten dazu bewegen, weniger Fleisch zu essen?

Studierende des UBRM lernen die Konzepte und Formen nachhaltigen Konsums kennen sowie die Herausforderungen in der Veränderung von Konsumverhalten zu verstehen, um daraus mögliche Handlungsoptionen für Unternehmen, Institutionen sowie für Forschung und Politik ableiten zu können. Sie erwerben im Bachelorstudium Grundlagenwissen über die Vermarktung von nachhaltigen Produkten und Dienstleistungen und können dieses in den Themenfeldern Konsumentenverhalten und marktorientierte Innovationen im Masterstudium vertiefen. Abschnitt 2.3.2 liefert 
erste Einblicke in das Thema nachhaltiger Konsum, dessen Ausgestaltungsmöglichkeiten und bekannte Hindernisse.

\subsubsection{Soziale, ökologische und ökonomische Perspektiven nachhaltigen Konsums}

Die sogenannte „Oslo-Definition“ liefert eine gängige Begriffserklärung für nachhaltigen Konsum. Sie wurde im Rahmen eines Symposiums zu nachhaltiger Produktion und nachhaltigem Konsum bereits 1994 formuliert. Nachhaltiger Konsum beschreibt demnach „... die Nutzung von Gütern und Dienstleistungen, die elementare menschliche Bedürfnisse befriedigen und eine bessere Lebensqualität hervorbringen, wobei sie gleichzeitig den Einsatz natürlicher Ressourcen, toxischer Stoffe und Emissionen von Abfall und Schadstoffen über den Lebenszyklus hinweg minimieren, um nicht die Bedürfnisbefriedigung künftiger Generationen zu gefährden" (Norwegisches Umweltministerium, zitiert nach Spangenberg 2003, S. 124).

In der Definition sind im Besonderen drei Aspekte entscheidend, nämlich (1) die elementare Bedürfnisbefriedigung, (2) die Verbesserung der Lebensqualität und (3) die Ressourcenschonung. Der erste Aspekt meint, dass weltweit in einer Weise und einem Ausmaß konsumiert werden soll, dass die Lebensgrundlage für Menschen (Ernährung, Wasser etc.) auf allen Teilen der Erde gesichert bleibt (oder wird). Für westliche Gesellschaften, in denen über die Deckung der Lebensgrundlage/Bedürfnisse und über ökologische Grenzen hinaus konsumiert wird (Druckman und Jackson 2010), stellt sich hier die Frage, welches Konsumausmaß für ein gutes Leben ausreicht, damit ökologische Grenzen gewahrt bleiben. Der zweite Aspekt kann sowohl ökologisch (z.B. saubere Luft), sozial (z.B. faire Arbeitsbedingungen) als auch wirtschaftlich (z.B. sicheres Einkommen) betrachtet werden. Diese drei Bedingungen spiegeln die drei Säulen der Nachhaltigkeit (siehe Abbildung 2.3.2) wider. Der dritte Aspekt ist als Bringschuld für künftige Generationen zu verstehen. Die Definition impliziert die Rolle der Akteurinnen und Akteure, insbesondere der Konsumentinnen und Konsumenten, durch aktuelles Handeln einen notwendigen Beitrag zu diesen Aspekten zu leisten.

Die Definition wählt hierbei den Begriff der Nutzung, der im engeren Sinn wohl zu kurz greift. Nachhaltiger Konsum sollte über die Nutzung hinaus auch als Kauf, Besitz und Nachnutzung verstanden werden. Für eine nachhaltige Entwicklung werden alternative Konsumformen zum Erwerb von Besitz (etwa Teilnutzung, sharing) gebraucht (Schanes et al. 2016). Der Erwerb von Besitz an Produkten stellt in westlichen Gesellschaften jedoch weiterhin den Normalfall dar. Es geht neben der Substitution von nichtnachhaltigen Waren und Dienstleistungen durch umwelt- und sozialverträgliche Alternativen auch um die Verstärkung alternativer Konsumformen. Die 


\section{Nachhaltiges Wirtschaften \& Unternehmensmanagement}

Nachnutzung durch Weitergabe oder Wiederverwertung ist ebenfalls unter Nutzung zu subsumieren. Die Konsumentinnen und Konsumenten spielen eine wesentliche Rolle in der Kreislaufwirtschaft (etwa durch fachgerechte Entsorgung von wiederverwertbaren Rohstoffen).

\subsubsection{Formen nachhaltigen Konsums}

Basierend auf dem beschriebenen Konzept des nachhaltigen Konsums (Abbildung 2.3.2) können Konsumentinnen und Konsumenten in unterschiedlichen Formen zu einer nachhaltigen Konsumentwicklung beitragen.

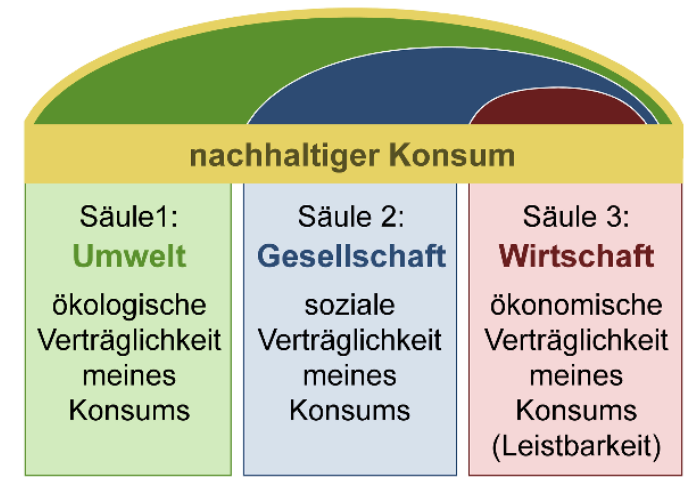

Abbildung 2.3.2: Drei-Säulen-Modell des nachhaltigen Konsums

Dabei lassen sich drei grundlegende Ansätze unterscheiden:

- Substitution („etwas anderes kaufen“, z.B. Wechsel zu effizienteren und/oder langlebigeren Produkten),

- Reduktion („weniger kaufen“, z.B. Wiederverwendung oder gemeinschaftliche Verwendung von Produkten),

- Verzicht („nicht kaufen“, z.B. gänzlicher Verzicht auf bestimmte Produkte und Dienstleistungen).

Bei der Substitution werden konventionelle Produkte und Dienstleistungen durch umwelt- und sozialverträglichere Alternativen ersetzt. Dies umfasst exemplarisch die Wahl von Produkten, die Rohstoffe effizienter nutzen (etwa weniger Treibstoff oder Strom benötigen) oder die aus nachwachsenden Rohstoffen hergestellt sind. Auf dem Gebiet der ökologischen Innovation wird aktuell viel Material- und Prozessforschung betrieben, um im Sinne einer Bioökonomie Produkte auf Basis nachwachsender Rohstoffe zu entwickeln. Zu nachhaltigem Konsum zählt aber ebenso der bewusste Verzicht auf Produkte von Unternehmen, die ihrer gesellschaftlichen Verantwortung nicht nachkommen. 
Reduktion bedeutet, dass weniger Rohstoffe abgebaut werden bzw. Waren im Umlauf sind (z.B. gemeinschaftliche Nutzung durch mehrere Haushalte; längere Verwendung durch Reparatur, Weiterverkauf oder Spenden; Wiederverwertung von Rohstoffen in einer Kreislaufwirtschaft). Fallbeispiel 2.3.2 beschreibt ein junges Wiener Unternehmen, das den Abfall von Lebensmitteln (somit am Ende der Wertschöpfungskette) zu vermeiden sucht, indem diese Lebensmittel weitergegeben und zu neuen Produkten verarbeitet werden. Eine ähnliche Initiative in Deutschland (Foodsharing.de) sammelt in Kooperation mit Betrieben und ehrenamtlichen Unterstützerinnen und Unterstützern Lebensmittelabfälle. Zur freien Entnahme stehen diese in Form von „Fair-Teilern“, d.h. öffentlich frei zugänglichen Lebensmittelregalen und Kühlschränken, zur Verfügung. Laut Betreiber konnten auf diese Weise knapp 22.000 Tonnen an Lebensmittelabfällen in Deutschland, Österreich und der Schweiz vermieden werden (Foodsharing 2019).

\section{Fallbeispiel 2.3.2: Unverschwendet - Eine Initiative als Beitrag zu nachhaltigem Lebensmittelkonsum}

\begin{tabular}{|c|c|}
\hline $\begin{array}{l}\text { UNVERSCHWENDET } \\
\text { Gutes aus gerettetem Obst und Gemüse. } \\
\text { ๑ Unverschwendet } \\
\text { gegründet im Jahr } 2015 \text { von } \\
\text { sich der Minimierung dieser L } \\
\text { Ansätze verschrieben. Das Wi } \\
\text { und Gemüse aus einem Netz } \\
\text { Es verkocht diese Lebensmitte } \\
\text { den im Jahr } 2018 \text { auf diese W } \\
\text { (Unverschwendet 2019). }\end{array}$ & $\begin{array}{l}\text { Österreichische Haushalte werfen jährlich mehrere Millionen Ton- } \\
\text { nen an genießbarem Obst und Gemüse in den Müll. Lebensmittel, } \\
\text { die qualitativ einwandfrei, jedoch aus Sicht der Verbraucherinnen } \\
\text { und Verbraucher zu groß, zu klein, zu unschön sind oder einfach } \\
\text { nicht aufgegessen wurden. Das junge Unternehmen „Unverschwendet", } \\
\text { der UBRM-Absolventin Cornelia Diesenreiter und ihrem Bruder, hat } \\
\text { ebensmittelabfälle (unter dem Stichwort zero waste) durch kreative } \\
\text { iener Start-up bezieht nichtverkäufliches oder nichtverbrauchtes Obst } \\
\text { verk von landwirtschaftlichen Betrieben, Institutionen und Haushalten. } \\
\text { el zu Marmeladen, Sirup und Saucen. } 25 \text { Tonnen Lebensmittel wur- } \\
\text { 'eise verarbeitet statt entsorgt und } 100.000 \text { Feinkostprodukte verkauft }\end{array}$ \\
\hline
\end{tabular}

Verzicht stellt das höchste Maß an Reduktion dar. Es bedeutet die bewusste Entscheidung, Waren und Dienstleistungen, die ökologisch oder sozial nicht verträglich sind, nicht zu konsumieren. Aktuell fokussieren die öffentliche Debatte und innovative Geschäftsideen vordergründig auf Formen der Substitution und Reduktion. Die Wichtigkeit des Konsumverzichts zur Erreichung von Klima- und Nachhaltigkeitszielen ist jedoch augenmerklich und findet sich beispielsweise in der aktuellen Strategie der Bioökonomie der österreichischen Regierung wieder (BMNT et al. 2019).

\subsubsection{Herausforderungen in der Entwicklung nachhaltigen Konsumverhaltens}

Konsumentinnen und Konsumenten können unterschiedliche Beiträge zur Erreichung des SDG 12 leisten. Das aktuelle Marktgeschehen und die Statistiken zum 


\section{Nachhaltiges Wirtschaften \& Unternehmensmanagement}

Ressourcenverbrauch von Haushalten zeigen aber, dass Veränderungen nicht so rasch voranschreiten, wie sie es müssten. Umfragen zeigen, dass die meisten Konsumentinnen und Konsumenten Klimaschutz und soziale Gerechtigkeit als wichtig erachten. Am Point of Sale vergessen sie jedoch ihre guten Vorsätze. Häufig spielen Produkteigenschaften - wie Marke, Preis oder Genuss - eine größere Rolle. Eine Metaanalyse basierend auf 57 empirischen Studien zeigt, dass die Einstellung zum Umweltschutz tatsächliches Verhalten nur zum Teil erklärt (vgl. Bamberg und Möser 2007). Diese Ergebnisse verdeutlichen, dass neben einer Bewusstseinsbildung für nachhaltige Themen auch andere Maßnahmen zur Entwicklung nachhaltiger Konsummuster ergriffen werden müssen.

Die Diskrepanz zwischen Bewusstsein, Werten und Einstellungen auf der einen Seite und dem tatsächlichen Handeln auf der anderen Seite wird als Attitude-BehaviourGap (oder Value-Action-Gap) bezeichnet (vgl. Kollmuss und Agyeman 2002). Der Abgleich von dem, was man tun möchte, und dem, wie man tatsächlich handelt, stellt eine der größten Herausforderungen bei der Vermeidung ressourcenintensiver sowie sozial unverträglicher Konsummuster dar (siehe Abbildung 2.3.3).

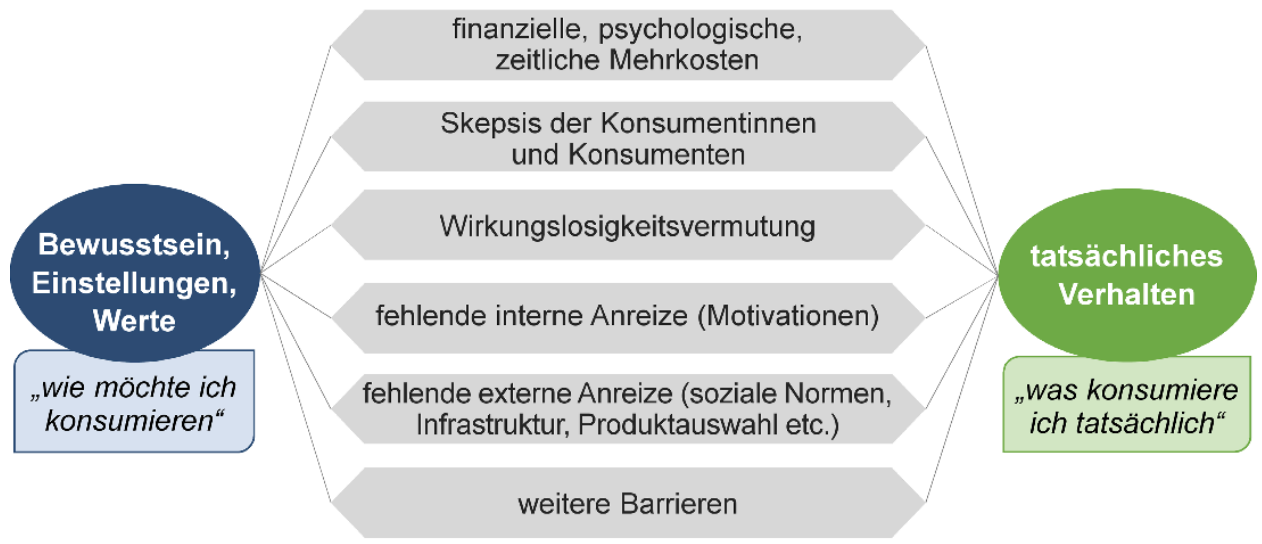

\section{Abbildung 2.3.3: Attitude-Behaviour-Gap und einige bekannte Barrieren}

Was hält Konsumentinnen und Konsumenten also davon ab, ihre guten Intentionen in die Tat umzusetzen? Die Barrieren sind mannigfaltig und umfassen z.B. finanzielle, psychologische wie zeitliche Mehrkosten für nachhaltige Alternativen; das Fehlen von internen Anreizen (Motivationen), von sozialen Normen, von positivem Feedback für nachhaltiges Verhalten und von notwendiger Infrastruktur; die Problematik von Konsumentenskepsis bezüglich Funktionalität und Informationswahrheit nachhaltiger Produkte als auch eine Wirkungslosigkeitsvermutung aufseiten der Konsumentinnen und Konsumenten (vgl. Kollmuss und Agyeman 2002). 
In Bezug auf Mehrkosten ist festzuhalten, dass sich Konsumentinnen und Konsumenten typischerweise für jene Produktalternative entscheiden, die ihnen den höchsten Produktwert (Gesamtnutzen minus Gesamtkosten) bietet. Der Konsum nachhaltiger Produkte hat neben dem gängigen funktionalen auch einen sozialen (altruistischen) und psychologischen Nutzen (man tut etwas „Gutes"). Nachhaltige Alternativen haben gleichzeitig häufig höhere wahrgenommene Kosten. Es entstehen (1) Suchkosten (Zeitaufwand) für das Finden, Verstehen und Berücksichtigen zusätzlicher Kriterien, die häufig aus Sicht der Konsumentinnen und Konsumenten schwierig zu beurteilen sind (z.B. faire Löhne in der Wertschöpfungskette, Tierwohl, ressourcenschonende Produktion). Unternehmen verwenden eine Vielzahl an Produktlabels zu Informationszwecken (siehe Beitrag 2.2). Diese werden jedoch nur von Teilen der Konsumentinnen und Konsumenten verstanden bzw. in die Kaufentscheidung miteinbezogen. Des Weiteren verursachen nachhaltige Alternativen häufig (2) finanzielle Mehrkosten oder (3) psychologische Kosten etwa durch die vorausgesetzte Änderung von Gewohnheiten. Menschen ändern ihre Gewohnheiten nur langsam und ungern, was etwa im anfänglichen Beispiel des Fleischkonsums eine wesentliche Barriere darstellt. Zu erwähnen sind auch Zielkonflikte zwischen Motiven (z.B. die Bequemlichkeit einer Take-away-Speise versus Vermeidung von Verpackungsabfall) und Trade-offs zwischen Produkteigenschaften (z.B. Leistungsstärke versus Nachhaltigkeit).

Die Skepsis der Konsumentinnen und Konsumenten gründet meist nicht in mangelnder Informationsbereitstellung vonseiten der Unternehmen zur Nachhaltigkeit ihrer Produkte und Dienstleistungen, sondern vielmehr darin, dass sie diesen Informationen nur bedingt Glauben schenken. Die zugrunde liegende Motivation von Unternehmen für soziales oder philanthropisches Engagement wird v.a. in europäischen Ländern kritisch hinterfragt. Da den Konsumentinnen und Konsumenten die Erfahrung mit Materialund Prozessinnovationen fehlt, besteht häufig auch Skepsis in Bezug auf die Funktionalität und Haltbarkeit von alternativen Materialien, die mit jenen in konventionellen Produkten verglichen werden.

In Bezug auf die Wirkungslosigkeitsvermutung erachtet die/der Einzelne ihren/seinen Beitrag zum nachhaltigen Konsum - aufgrund der Größe des Problems - als nutzlos. Das eigene Zutun wird daher (vor dem Gesichtspunkt der zusätzlichen Kosten) als vernachlässigbar eingeschätzt und nicht verfolgt.

\subsubsection{Ausblick: Nachhaltiger Maßnahmenmix und sein Beitrag}

Der Dringlichkeit, ressourcenintensives Konsumverhalten zu verändern, steht somit eine Reihe an Hindernissen gegenüber, die einer breiten Durchsetzung von nachhaltigen Konsummustern entgegenwirken. Wie Abbildung 2.3.4 illustriert, agieren die 


\section{Nachhaltiges Wirtschaften \& Unternehmensmanagement}

Konsumentinnen und Konsumenten als individuelle Wesen in einem sozialen, kulturellen und politischen Umfeld, das ihr Verhalten beeinflusst und somit förderlich auf nachhaltiges Konsumieren wirken kann. Die Konsumverhaltensforschung vereint Theorien und Werkzeuge zahlreicher Disziplinen (Verhaltensökonomik, Psychologie, Soziologie), um effektive Maßnahmen zur Beseitigung oder Entkräftung solcher Hindernisse zu identifizieren. Positive Anreize (gesellschaftliche Wertschätzung, finanzielle Subventionen) erhöhen den erzielten Nutzen bei Wahl einer nachhaltigen Konsumalternative. Die Schaffung und Weiterentwicklung geeigneter Institutionen und Rahmenbedingungen fördern nachhaltigen Konsum (vgl. Beitrag 5.3). Gebote und Verbote (z.B. Beschluss der EU zum Verbot von Einwegplastik ab dem Jahr 2021 (Richtlinie über Einwegkunststoffe 2019)) unterstützen eine effektive Verhaltenssteuerung. Maßnahmen wie Green Nudging versuchen hingegen, eine Verhaltensänderung ohne Verbote, Gebote oder eine Veränderung der Anreize zu erwirken (vgl. Thaler und Sunstein 2008).

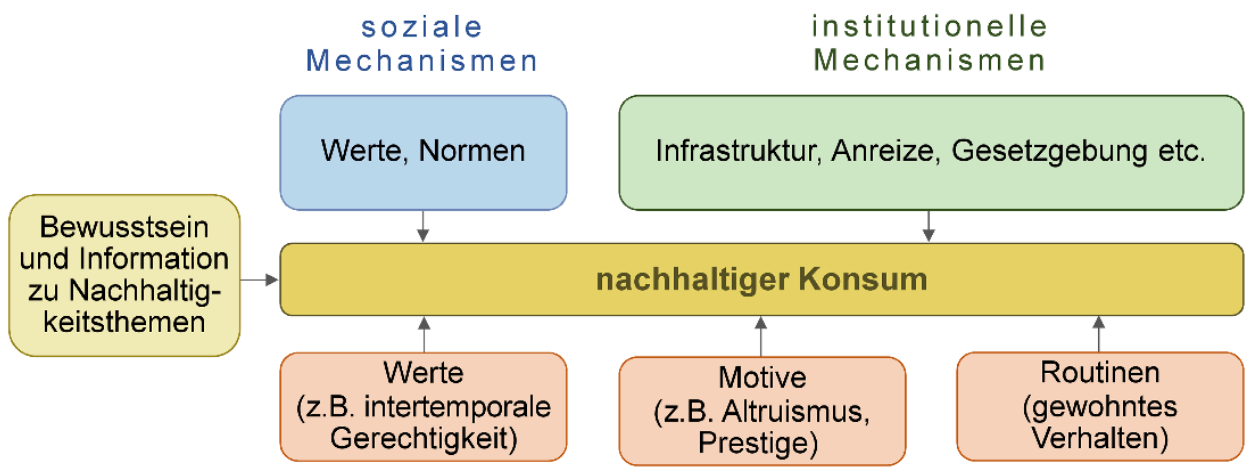

individuelle Mechanismen

\section{Abbildung 2.3.4: Einflussgrößen auf das Konsumverhalten}

Dieses Zusammenspiel an Einflussgrößen zeigt deutlich, es bedarf einer gemeinsamen und weltweiten Anstrengung von Konsumentinnen und Konsumenten, Unternehmen und der Politik, um die Ziele des SDG 12 schrittweise zu erreichen. Ein erster Schritt wäre die Substitution von umweltschädlichen Konsumoptionen durch nachhaltige. Langfristig bedarf es einer gesellschaftlichen Debatte und Neuorientierung, was suffizienter (maßvoller) Konsum beitragen kann, um die Bedürfnisse gegenwärtiger und zukünftiger Konsumentinnen und Konsumenten in Einklang zu bringen. Gegenwärtig kann maßvoller Konsum in den reichen Teilen der Welt einen Beitrag zur Armuts- und Hungerbekämpfung leisten (SDG 1 und 2), weil Ressourcen so gerechter verteilt werden können.

UBRM-Studierende lernen, Zusammenhänge zwischen den drei Säulen des nachhaltigen Konsums herzustellen sowie Konsumentscheidungen und Veränderungsmechanis- 
men zu verstehen. Diese Kenntnisse befähigen sie, in Betrieben und Organisationen an nachhaltigen Gesellschafts- und Wirtschaftskonzepten mitzuarbeiten.

\section{Literatur}

Bamberg, S. and Möser, G. (2007): Twenty years after Hines, Hungerford, and Tomera: A new meta-analysis of psycho-social determinants of pro-environmental behaviour. Journal of Environmental Psychology, 27, 1, 14-25. https://doi.org/10.1016/J.JENVP.2006.12.002.

BMNT, BMVIT und BMBWF (Bundesministerium für Nachhaltigkeit und Tourismus; Bundesministerium für Bildung, Wissenschaft und Forschung; Bundesministerium für Verkehr, Innovation und Technologie) (2019): Bioökonomie - Eine Strategie für Österreich. Wien. Verfügbar in: https://www.bmnt.gv.at/umwelt/klimaschutz/biooekonomie/Bio\%C3\%B6konomieStrategie-f\%C3\%BCr-\%C3\%96sterreich.html [Abfrage am 1.4.2019].

Druckman, A. and Jackson, T. (2010): The bare necessities: How much household carbon do we really need? Ecological Economics, 69, 9, 1794-1804. https://doi.org/10.1016/J.ECOLECON.2010.04.018.

Foodsharing (2019): Foodsharing Statistik. Verfügbar in: https://foodsharing.de/statistik [Abfrage am 24.3.2019].

Hertwich, E. G., Vita, G., Wood, R., Ivanova, D., Tukker, A., Stadler, K., and Steen-Olsen, K. (2015): Environmental impact assessment of household consumption. Journal of Industrial Ecology, 20, 3, 526-536. https://doi.org/10.1111/jiec.12371.

Kollmuss, A. and Agyeman, J. (2002): Mind the gap: Why do people act environmentally and what are the barriers to pro-environmental behavior? Environmental Education Research, 8, 3, 239-260. https://doi.org/10.1080/13504620220145401.

Richtlinie über Einwegkunststoffe (2019): Richtlinie (EU) 2019/904 des Europäischen Parlaments und des Rates vom 5. Juni 2019 über die Verringerung der Auswirkungen bestimmter Kunststoffprodukte auf die Umwelt. Abl L 155/2019, 1. Verfügbar in: http://data.europa.eu/eli/dir/2019/904/oj [Abfrage am 20.7.2019].

Riefler, P. (2019, in press): Consumer responses to MNE socially-responsible behavior. In: Leonidou, L. C., Katsikeas, C. S., Samiee, S., Leonidou, C. N., eds., Socially Responsible International Business: Critical Issues and the Way Forward. Cheltenham: Edward Elgar Publishing.

Schanes, K., Giljum, S., and Hertwich, E. (2016). Low carbon lifestyles: A framework to structure consumption strategies and options to reduce carbon footprints. Journal of Cleaner Production, 139, 1033-1043. https://doi.org/10.1016/j.jclepro.2016.08.154.

Spangenberg, J. (2003): Vision 2020: Arbeit, Umwelt, Gerechtigkeit: Strategien für ein zukunftsfähiges Deutschland. München: ökom Verlag.

Thaler, R. H. and Sunstein, C. R. (2008): Nudge: Improving Decisions about Health, Wealth, and Happiness. New Haven, London: Yale University Press.

UN (United Nations) (2016): Transforming our world: the 2030 Agenda for Sustainable Development. Sustainable Development Knowledge Platform. Sustainable Development Goals. Available at: https://sustainabledevelopment.un.org/post2015/transformingourworld [accessed 1.4.2019].

Unverschwendet (2019): Verfügbar in: https://www.unverschwendet.at/ [Abfrage am 4.4.2019].

WWF (Worldwide Fund For Nature) (2019): Bio, Gesund und Leistbar. Ja, das geht! Umweltfreundliche und gesunde Ernährung in Österreich. Verfügbar in:

https://www.wwf.at/de/view/files/download/showDownload/?tool=12\&feld=download\& sprach connect=3351 [Abfrage am 1.4.2019]. 
Open Access Dieses Kapitel wird unter der Creative Commons Namensnennung - Nicht kommerziell 4.0 International Lizenz (http://creativecommons.org/licenses/by-nc/4.0/deed.de)veröffentlicht, welche die nicht-kommerzielle Nutzung, Vervielfältigung, Bearbeitung, Verbreitung und Wiedergabe in jeglichem Medium und Format erlaubt, sofern Sie den/die ursprünglichen Autor(en) und die Quelle ordnungsgemäß nennen, einen Link zur Creative Commons Lizenz beifügen und angeben, ob Änderungen vorgenommen wurden.

Die in diesem Kapitel enthaltenen Bilder und sonstiges Drittmaterial unterliegen ebenfalls der genannten Creative Commons Lizenz, sofern sich aus der Abbildungslegende nichts anderes ergibt. Sofern das betreffende Material nicht unter der genannten Creative Commons Lizenz steht und die betreffende Handlung nicht nach gesetzlichen Vorschriften erlaubt ist, ist auch für die oben aufgeführten nicht-kommerziellen Weiterverwendungen des Materials die Einwilligung des jeweiligen Rechteinhabers einzuholen.

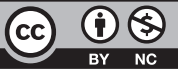

